\title{
Some New Coupled Coincidence Point and Coupled Fixed Point Results in Partially Ordered Metric-Like Spaces and an Application
}

\author{
Min Liang $(\mathbb{D}$, Chuanxi Zhu $\mathbb{D}$, Zhaoqi Wu, and Chunfang Chen \\ Department of Mathematics, Nanchang University, Nanchang 330031, China \\ Correspondence should be addressed to Chuanxi Zhu; chuanxizhu@126.com
}

Received 28 March 2018; Revised 9 July 2018; Accepted 15 July 2018; Published 1 August 2018

Academic Editor: Ismat Beg

Copyright (c) 2018 Min Liang et al. This is an open access article distributed under the Creative Commons Attribution License, which permits unrestricted use, distribution, and reproduction in any medium, provided the original work is properly cited.

Some new coupled coincidence point and coupled fixed point theorems are established in partially ordered metric-like spaces, which generalize many results in corresponding literatures. An example is given to support our main results. As an application, we discuss the existence of the solutions for a class of nonlinear integral equations.

\section{Introduction and Preliminaries}

As we all know, the fixed point theory is one of the most important tools in the field of nonlinear analysis. In particular, the coupled fixed point theorems in partially ordered metric-like spaces are very valuable for discussing the existence and uniqueness of solutions of any nonlinear problem in fields of mathematics and physics.

In order to solve the more complex nonlinear analysis problems, the concept of a metric space has been extended in many aspects. In 1992, the notion of a partial metric space was introduced by Matthews [1]. Considering $d(x, x)$ may be greater than zero, he tried to extend the concept of a metric space. Since then, many authors proved various fixed point theorems in partial metric spaces (see [2-9]). In the interest of studying the existence of solutions of ordinary differential equations, the mixed monotone mapping was established. Afterward, Lakshmikantham and Ćirić [10] substituted the mixed $g$-monotone mapping for the mixed monotone mapping and proved the coupled common fixed point theorems. On the other hand, Hitzler and Seda [11] first studied the dislocated space in 2000, and then AminiHarandi [12] named it as a metric-like space. Subsequently, some authors discussed the fixed point and coincidence point results in generalized metric spaces. And several applications to operator equations and integral equations are given in a line of research (see [13-19]). Recently, B. Hazarika et al. [20] generalized some previous results and gave some new common fixed point theorems in metric-like spaces.

In this paper, inspired by the above literatures, we propose some new coupled coincidence point and coupled fixed point theorems in partially ordered metric-like spaces, which extend the theorems of B. Hazarika et al. [20]. As an application, we discuss the existence of solutions for a system of nonlinear integral equations to illustrate our main results.

First, we review some concepts which are going to be used later.

Throughout this paper, let $\mathbb{R}=(-\infty,+\infty), \mathbb{R}^{+}=$ $(0,+\infty)$, and $\mathbb{R}_{0}^{+}=[0,+\infty)$. Let $\mathbb{N}$ be the set of all natural numbers and $\mathbb{N}^{+}$be the set of all positive integer numbers.

Definition 1 (see [12]). Let $X$ be a nonempty set. A function $\sigma: X \times X \longrightarrow \mathbb{R}_{0}^{+}$is said to be a dislocated (metric-like) metric on $X$ if, for all $x, y, z \in X$, the following conditions hold:

$\left(\sigma_{1}\right) \sigma(x, y)=0 \Longrightarrow x=y$.

$\left(\sigma_{2}\right) \sigma(x, y)=\sigma(y, x)$.

$\left(\sigma_{3}\right) \sigma(x, y) \leq \sigma(x, z)+\sigma(z, y)$.

Definition 2 (see [8]). Let $(X, \sigma)$ be a metric-like space.

(a) A sequence $\left\{x_{n}\right\}$ in $X$ is said to be a Cauchy sequence if $\lim _{n, m \rightarrow \infty} \sigma\left(x_{n}, x_{m}\right)$ exists and is finite. 
(b) $(X, \sigma)$ is said to be complete if every Cauchy sequence $\left\{x_{n}\right\}$ in $X$ converges with respect to $\tau_{\sigma}$ to a point $x \in X$ such that

$$
\lim _{n \longrightarrow \infty} \sigma\left(x_{n}, x\right)=\sigma(x, x)=\lim _{n, m \longrightarrow \infty} \sigma\left(x_{n}, x_{m}\right) .
$$

Definition 3 (see [6]). Let $\psi, \phi:[0, \infty) \longrightarrow[0, \infty)$ be two functions. A pair of functions $(\psi, \phi)$ is said to belong to the class $\Psi$ if they satisfy the following conditions:

(i) For $u, v \in[0,+\infty)$ if $\psi(u) \leq \phi(v)$, then $u \leq v$.

(ii) For $u_{n}, v_{n} \in[0,+\infty)$ with $\lim _{n \longrightarrow \infty} u_{n}=\lim _{n \rightarrow \infty} v_{n}=$ $w$, if $\psi\left(u_{n}\right) \leq \phi\left(v_{n}\right)$ for all $n$, then $w=0$.

Definition 4 (see [7]). Let $\mathbb{F}$ be the class of all functions $F$ : $[0, \infty)^{3} \longrightarrow[0, \infty)$ satisfying the following conditions:

$\left(F_{1}\right) \max \{a, b\} \leq F(a, b, c)$ for all $a, b, c \geq 0$.

$\left(F_{2}\right) F(a, 0,0)=a$ for all $a \geq 0$.

$\left(F_{3}\right) F$ is continuous.

Definition 5 (see [9]). Let $(X, \preceq)$ be a partially ordered set and $T: X \times X \longrightarrow X$. Then the map $T$ is said to have mixed monotone property if $T(x, y)$ is monotone nondecreasing in $x$ and is monotone nonincreasing in $y$; that is,

$$
\begin{array}{ll}
x_{1} \preceq x_{2} \text { implies } T\left(x_{1}, y\right) \preceq T\left(x_{2}, y\right) & \text { for all } y \in X, \\
y_{1} \preceq y_{2} \text { implies } T\left(x, y_{2}\right) \preceq T\left(x, y_{1}\right) & \text { for all } x \in X .
\end{array}
$$

Definition 6 (see $[10])$. Let $(X, \preceq)$ be a partially ordered set and $T: X \times X \longrightarrow X$. Then the map $T$ is said to have mixed gmonotone property if $T(x, y)$ is monotone g-nondecreasing in $x$ and is monotone $\mathrm{g}$-nonincreasing in $y$; that is,

$$
\begin{aligned}
& \mathrm{g} x_{1} \preceq \mathrm{g} x_{2} \text { implies } T\left(x_{1}, y\right) \preceq T\left(x_{2}, y\right) \\
& \text { for all } y \in X,
\end{aligned}
$$$$
\mathrm{g} y_{1} \preceq \mathrm{g} y_{2} \text { implies } T\left(x, y_{2}\right) \preceq T\left(x, y_{1}\right)
$$

for all $x \in X$.

Definition 7 (see [9]). An element $(x, y) \in X \times X$ is called a coupled fixed point for the mapping $T: X \times X \longrightarrow X$ if $T(x, y)=x$ and $T(y, x)=y$.

Definition 8 (see [10]). Let $X$ be a nonempty set. We say that the mappings $T: X \times X \longrightarrow X$ and $\mathrm{g}: X \longrightarrow X$ are commutative if $g T(x, y)=T(\mathrm{~g} x, \mathrm{~g} y)$, for all $x, y \in X$.

Definition 9 (see [10]). An element $(x, y) \in X \times X$ is called a coupled coincidence point of the mapping $T: X \times X \longrightarrow X$ and $\mathrm{g}: X \longrightarrow X$ if $T(x, y)=\mathrm{g} x$ and $T(y, x)=\mathrm{g} y$.

\section{Main Results}

In this section, we put forward a new coupled coincidence point theorem in a partially ordered metric-like space, and if $\mathrm{g}=I$, which is a self-mapping, we get a new coupled fixed point theorem. Then, we establish a common coupled fixed point theorem in a partially ordered metric-like space and prove the uniqueness of the coupled fixed point.
Theorem 10. Let $(X, \preceq)$ be a partially ordered set and $(X, \sigma)$ be a complete metric-like space. Let $\mathrm{T}: X \times X \longrightarrow X$ and $\mathrm{g}$ : $X \longrightarrow X$ be two mappings such that the following conditions are satisfied:

(i) $\mathrm{T}(X \times X) \subseteq \mathrm{g}(X)$.

(ii) $\mathrm{g}(X)$ is closed.

(iii) $T$ has the mixed g-monotone property.

(iv) There exist $x_{0}, y_{0} \in X$ such that $\mathrm{g} x_{0} \preceq T\left(x_{0}, y_{0}\right)$ and $T\left(y_{0}, x_{0}\right) \preceq \mathrm{g} y_{0}$.

(v)

$$
\begin{aligned}
& \psi(F(\sigma(T(x, y), T(u, v)), \varphi(T(x, y)), \varphi(T(u, v)))) \\
& \quad \leq \phi(M(x, u, y, v))
\end{aligned}
$$

where

$$
\begin{gathered}
M(x, u, y, v)=\max \{F(\sigma(\mathrm{g} x, \mathrm{~g} u), \varphi(\mathrm{g} x), \varphi(\mathrm{g} u)), \\
F(\sigma(\mathrm{g} y, \mathrm{~g} v), \varphi(\mathrm{g} y), \varphi(\mathrm{g} v)), \\
F(\sigma(\mathrm{g} x, T(x, y)), \varphi(\mathrm{g} x), \varphi(T(x, y))), \\
F(\sigma(\mathrm{g} y, T(y, x)), \varphi(\mathrm{g} y), \varphi(T(y, x)))\},
\end{gathered}
$$

with $\mathrm{g} x \preceq \mathrm{g} u$ and $\mathrm{g} v \preceq \mathrm{g} y$, or $\mathrm{g} u \preceq \mathrm{g} x$ and $\mathrm{g} y \preceq \mathrm{g} v$ for all $x, y, u, v \in X, F \in \mathbb{F},(\psi, \phi) \in \Psi, \psi$ is nondecreasing, and $\varphi: X \longrightarrow[0,+\infty)$ is a lower semicontinuous function.

Then $T$ and $g$ have a coupled coincidence point.

Proof. Let $x_{0}, y_{0} \in X$ such that $g x_{0} \preceq T\left(x_{0}, y_{0}\right)$ and $T\left(y_{0}, x_{0}\right) \preceq \mathrm{g} y_{0}$. Since $\mathrm{T}(X \times X) \subseteq \mathrm{g}(X)$, we can find $x_{1}, y_{1} \in$ $X$ such that $g x_{1}=T\left(x_{0}, y_{0}\right)$ and $g y_{1}=T\left(y_{0}, x_{0}\right)$. Similarly, there exist $x_{2}, y_{2} \in X$ such that $g x_{2}=T\left(x_{1}, y_{1}\right)$ and $g y_{2}=$ $T\left(y_{1}, x_{1}\right)$. Repeating the above process, we can construct two sequences $\left\{x_{n}\right\},\left\{y_{n}\right\}$ such that $g x_{n+1}=T\left(x_{n}, y_{n}\right)$ and $g y_{n+1}=$ $T\left(y_{n}, x_{n}\right)$. Since $T$ has the mixed g-monotone property, we get $\mathrm{g} x_{0} \preceq \mathrm{g} x_{1} \preceq \mathrm{g} x_{2} \preceq \cdots \preceq \mathrm{g} x_{n} \preceq \cdots$ and $\mathrm{g} y_{0} \succeq$ $\mathrm{g} y_{1} \geq \mathrm{g} y_{2} \geq \cdots \geq \mathrm{g} y_{n} \geq \cdots$. If for some $n \in \mathbb{N}$ and we have $\left(\mathrm{g} x_{n+1}, \mathrm{~g} y_{n+1}\right)=\left(\mathrm{g} x_{n}, \mathrm{~g} y_{n}\right)$, then $T\left(x_{n}, y_{n}\right)=\mathrm{g} x_{n+1}=$ $\mathrm{g} x_{n}, T\left(y_{n}, x_{n}\right)=\mathrm{g} y_{n+1}=\mathrm{g} y_{n}$. This means $T$ and $\mathrm{g}$ have $\mathrm{a}$ coupled coincidence point.

First, we show that if $T$ and $g$ have a coupled coincidence point, i.e., $T(x, y)=\mathrm{g} x, T(y, x)=\mathrm{g} y$, then $\sigma(\mathrm{g} x, \mathrm{~g} x)=$ $\sigma(T(x, y), T(x, y))=\sigma(\mathrm{g} y, \mathrm{~g} y)=\sigma(T(y, x), T(y, x))=0$. Indeed, we have $\mathrm{g} x \preceq \mathrm{g} x$, and supposing $\sigma(\mathrm{g} x, \mathrm{~g} x)>0$ and using (4), we obtain

$$
\begin{aligned}
\psi & (F(\sigma(\mathrm{g} x, \mathrm{~g} x), \varphi(\mathrm{g} x), \varphi(\mathrm{g} x))) \\
& =\psi(F(\sigma(T(x, y), T(x, y)), \varphi(T(x, y)), \\
& \varphi(T(x, y)))) \leq \phi(M(x, x, y, y)) \\
& =\phi(\max \{F(\sigma(\mathrm{g} x, \mathrm{~g} x), \varphi(\mathrm{g} x), \varphi(\mathrm{g} x)), \\
& F(\sigma(\mathrm{g} y, \mathrm{~g} y), \varphi(\mathrm{g} y), \varphi(\mathrm{g} y)), \\
& F(\sigma(\mathrm{g} x, T(x, y)), \varphi(\mathrm{g} x), \varphi(T(x, y))), \\
& F(\sigma(\mathrm{g} y, T(y, x)), \varphi(\mathrm{g} y), \varphi(T(y, x)))\}) \\
& =\phi(\max \{F(\sigma(\mathrm{g} x, \mathrm{~g} x), \varphi(\mathrm{g} x), \varphi(\mathrm{g} x)), \\
& F(\sigma(\mathrm{g} y, \mathrm{~g} y), \varphi(\mathrm{g} y), \varphi(\mathrm{g} y))\}) .
\end{aligned}
$$


Similarly, since gy gy, we get

$$
\begin{aligned}
& \psi(F(\sigma(\mathrm{g} y, \mathrm{~g} y), \varphi(\mathrm{g} y), \varphi(\mathrm{g} y)))=\psi(F(\sigma(T(y, x), \\
& T(y, x)), \varphi(T(y, x)), \varphi(T(y, x)))) \leq \phi(M(y, y, x, \\
& x))=\phi(\max \{F(\sigma(\mathrm{g} y, \mathrm{~g} y), \varphi(\mathrm{g} y), \varphi(\mathrm{g} y)), \\
& F(\sigma(\mathrm{g} x, \mathrm{~g} x), \varphi(\mathrm{g} x), \varphi(\mathrm{g} x)), \\
& F(\sigma(\mathrm{g} y, T(y, x)), \varphi(\mathrm{g} y), \varphi(T(y, x))), \\
& F(\sigma(\mathrm{g} x, T(x, y)), \varphi(\mathrm{g} x), \varphi(T(x, y)))\}) \\
& =\phi(\max \{F(\sigma(\mathrm{g} x, \mathrm{~g} x), \varphi(\mathrm{g} x), \varphi(\mathrm{g} x)), \\
& F(\sigma(\mathrm{g} y, \mathrm{~g} y), \varphi(\mathrm{g} y), \varphi(\mathrm{g} y))\}) .
\end{aligned}
$$

Noting that $\psi$ is nondecreasing and using (6) and (7), we have

$$
\begin{aligned}
& \psi(\max \{F(\sigma(\mathrm{g} x, \mathrm{~g} x), \varphi(\mathrm{g} x), \varphi(\mathrm{g} x)), \\
& F(\sigma(\mathrm{g} y, \mathrm{~g} y), \varphi(\mathrm{g} y), \varphi(\mathrm{g} y))\}) \\
& =\max \{\psi(F(\sigma(\mathrm{g} x, \mathrm{~g} x), \varphi(\mathrm{g} x), \varphi(\mathrm{g} x))), \\
& \psi(F(\sigma(\mathrm{g} y, \mathrm{~g} y), \varphi(\mathrm{g} y), \varphi(\mathrm{g} y)))\} \\
& \leq \phi(\max \{F(\sigma(\mathrm{g} x, \mathrm{~g} x), \varphi(\mathrm{g} x), \varphi(\mathrm{g} x)), \\
& F(\sigma(\mathrm{g} y, \mathrm{~g} y), \varphi(\mathrm{g} y), \varphi(\mathrm{g} y))\}) \text {. }
\end{aligned}
$$

By Definition 3, we obtain that if $\psi(w) \leq \phi(w)$, then $w=0$. On the other hand, through the definition of $F$, we can reach the following conclusions:

$$
\begin{gathered}
\max \{F(\sigma(\mathrm{g} x, \mathrm{~g} x), \varphi(\mathrm{g} x), \varphi(\mathrm{g} x)), \\
F(\sigma(\mathrm{g} y, \mathrm{~g} y), \varphi(\mathrm{g} y), \varphi(\mathrm{g} y))\}=0, \\
\max \{\sigma(\mathrm{g} x, \mathrm{~g} x), \sigma(\mathrm{g} y, \mathrm{~g} y), \varphi(\mathrm{g} x), \varphi(\mathrm{g} y)\} \\
\leq \max \{F(\sigma(\mathrm{g} x, \mathrm{~g} x), \varphi(\mathrm{g} x), \varphi(\mathrm{g} x)), \\
F(\sigma(\mathrm{g} y, \mathrm{~g} y), \varphi(\mathrm{g} y), \varphi(\mathrm{g} y))\}=0 .
\end{gathered}
$$

This is a contradiction. Hence, if there exist $x, y \in X$ such that $T(x, y)=g x, T(y, x)=g y$, we have

$$
\sigma(\mathrm{g} x, \mathrm{~g} x)=\sigma(\mathrm{g} y, \mathrm{~g} y)=\varphi(\mathrm{g} x)=\varphi(\mathrm{g} y)=0 .
$$

Now, suppose that $\left(\mathrm{g} x_{n+1}, \mathrm{~g} y_{n+1}\right) \neq\left(\mathrm{g} x_{n}, \mathrm{~g} y_{n}\right)$ for all $n \in \mathbb{N}$, which means that either $\mathrm{g} x_{n} \neq T\left(x_{n}, y_{n}\right)$ or $\mathrm{g} y_{n} \neq$ $T\left(y_{n}, x_{n}\right)$. Since $\mathrm{g} x_{n-1} \preceq \mathrm{g} x_{n}$ and $\mathrm{g} y_{n} \preceq \mathrm{g} y_{n-1}$, we obtain

$$
\begin{aligned}
& \psi\left(F\left(\sigma\left(g x_{n}, g x_{n+1}\right), \varphi\left(g x_{n}\right), \varphi\left(g x_{n+1}\right)\right)\right) \\
& \quad=\psi\left(F \left(\sigma\left(T\left(x_{n-1}, y_{n-1}\right), T\left(x_{n}, y_{n}\right)\right),\right.\right. \\
& \left.\left.\varphi\left(T\left(x_{n-1}, y_{n-1}\right)\right), \varphi\left(T\left(x_{n}, y_{n}\right)\right)\right)\right) \leq \phi\left(M \left(x_{n-1},\right.\right. \\
& \left.\left.\quad x_{n}, y_{n-1}, y_{n}\right)\right)
\end{aligned}
$$

where

$$
\begin{aligned}
& M\left(x_{n-1}, x_{n}, y_{n-1}, y_{n}\right)=\max \left\{F \left(\sigma\left(\mathrm{g} x_{n-1}, \mathrm{~g} x_{n}\right),\right.\right. \\
& \left.\varphi\left(\mathrm{g} x_{n-1}\right), \varphi\left(\mathrm{g} x_{n}\right)\right), F\left(\sigma\left(\mathrm{g} y_{n-1}, \mathrm{~g} y_{n}\right), \varphi\left(\mathrm{g} y_{n-1}\right),\right. \\
& \left.\varphi\left(\mathrm{g} y_{n}\right)\right), F\left(\sigma\left(\mathrm{g} x_{n-1}, T\left(x_{n-1}, y_{n-1}\right)\right), \varphi\left(\mathrm{g} x_{n-1}\right),\right. \\
& \left.\varphi\left(T\left(x_{n-1}, y_{n-1}\right)\right)\right), F\left(\sigma\left(\mathrm{g} y_{n-1}, T\left(y_{n-1}, x_{n-1}\right)\right),\right. \\
& \left.\left.\varphi\left(\mathrm{g} y_{n-1}\right), \varphi\left(T\left(y_{n-1}, x_{n-1}\right)\right)\right)\right\} \\
& =\max \left\{F\left(\sigma\left(\mathrm{g} x_{n-1}, \mathrm{~g} x_{n}\right), \varphi\left(\mathrm{g} x_{n-1}\right), \varphi\left(\mathrm{g} x_{n}\right)\right),\right. \\
& \left.F\left(\sigma\left(\mathrm{g} y_{n-1}, \mathrm{~g} y_{n}\right), \varphi\left(\mathrm{g} y_{n-1}\right), \varphi\left(\mathrm{g} y_{n}\right)\right)\right\} .
\end{aligned}
$$

Similarly, we have

$$
\begin{aligned}
\psi & \left(F\left(\sigma\left(\mathrm{g} y_{n}, \mathrm{~g} y_{n+1}\right), \varphi\left(\mathrm{g} y_{n}\right), \varphi\left(\mathrm{g} y_{n+1}\right)\right)\right) \\
& =\psi\left(F \left(\sigma\left(T\left(y_{n-1}, x_{n-1}\right), T\left(y_{n}, x_{n}\right)\right),\right.\right. \\
& \left.\left.\varphi\left(T\left(y_{n-1}, x_{n-1}\right)\right), \varphi\left(T\left(y_{n}, x_{n}\right)\right)\right)\right) \leq \phi\left(M \left(y_{n-1},\right.\right. \\
& \left.\left.y_{n}, x_{n-1}, x_{n}\right)\right),
\end{aligned}
$$

where

$$
\begin{aligned}
& M\left(y_{n-1}, y_{n}, x_{n-1}, x_{n}\right)=\max \left\{F \left(\sigma\left(\mathrm{g} y_{n-1}, \mathrm{~g} y_{n}\right),\right.\right. \\
& \left.\varphi\left(\mathrm{g} y_{n-1}\right), \varphi\left(\mathrm{g} y_{n}\right)\right), F\left(\sigma\left(\mathrm{g} x_{n-1}, \mathrm{~g} x_{n}\right), \varphi\left(\mathrm{g} x_{n-1}\right),\right. \\
& \left.\varphi\left(\mathrm{g} x_{n}\right)\right), F\left(\sigma\left(\mathrm{g} y_{n-1}, T\left(y_{n-1}, x_{n-1}\right)\right), \varphi\left(\mathrm{g} y_{n-1}\right),\right. \\
& \left.\varphi\left(T\left(y_{n-1}, x_{n-1}\right)\right)\right), F\left(\sigma\left(\mathrm{g} x_{n-1}, T\left(x_{n-1}, y_{n-1}\right)\right),\right. \\
& \left.\left.\varphi\left(\mathrm{g} x_{n-1}\right), \varphi\left(T\left(x_{n-1}, y_{n-1}\right)\right)\right)\right\} \\
& =\max \left\{F\left(\sigma\left(\mathrm{g} x_{n-1}, \mathrm{~g} x_{n}\right), \varphi\left(\mathrm{g} x_{n-1}\right), \varphi\left(\mathrm{g} x_{n}\right)\right),\right. \\
& \left.F\left(\sigma\left(\mathrm{g} y_{n-1}, \mathrm{~g} y_{n}\right), \varphi\left(\mathrm{g} y_{n-1}\right), \varphi\left(\mathrm{g} y_{n}\right)\right)\right\} .
\end{aligned}
$$

Noting that $\psi$ is nondecreasing, using (11) and (13) we get

$$
\begin{aligned}
& \psi\left(\operatorname { m a x } \left\{F\left(\sigma\left(\mathrm{g} x_{n}, \mathrm{~g} x_{n+1}\right), \varphi\left(\mathrm{g} x_{n}\right), \varphi\left(\mathrm{g} x_{n+1}\right)\right),\right.\right. \\
& \left.\left.\quad F\left(\sigma\left(\mathrm{g} y_{n}, \mathrm{~g} y_{n+1}\right), \varphi\left(\mathrm{g} y_{n}\right), \varphi\left(\mathrm{g} y_{n+1}\right)\right)\right\}\right) \\
& =\max \left\{\psi\left(F\left(\sigma\left(\mathrm{g} x_{n}, \mathrm{~g} x_{n+1}\right), \varphi\left(\mathrm{g} x_{n}\right), \varphi\left(\mathrm{g} x_{n+1}\right)\right)\right),\right. \\
& \psi \\
& \left.\quad \leq\left(F\left(\sigma\left(\mathrm{g} y_{n}, \mathrm{~g} y_{n+1}\right), \varphi\left(\mathrm{g} y_{n}\right), \varphi\left(\mathrm{g} y_{n+1}\right)\right)\right)\right\} \\
& \quad \\
& \quad F\left(\sigma\left(\mathrm{gax}\left\{F\left(\sigma\left(\mathrm{g} x_{n-1}, \mathrm{~g} y_{n}\right), \varphi\left(\mathrm{g} x_{n}\right), \varphi\left(\mathrm{g} y_{n-1}\right), \varphi\left(\mathrm{g} x_{n-1}\right)\right)\right\}\right) .\right.
\end{aligned}
$$

Using condition (i) of Definition 3, we get

$$
\begin{aligned}
& \max \left\{F\left(\sigma\left(\mathrm{g} x_{n}, \mathrm{~g} x_{n+1}\right), \varphi\left(\mathrm{g} x_{n}\right), \varphi\left(\mathrm{g} x_{n+1}\right)\right),\right. \\
& \left.\quad F\left(\sigma\left(\mathrm{g} y_{n}, \mathrm{~g} y_{n+1}\right), \varphi\left(\mathrm{g} y_{n}\right), \varphi\left(\mathrm{g} y_{n+1}\right)\right)\right\} \\
& \quad \leq \max \left\{F\left(\sigma\left(\mathrm{g} x_{n-1}, \mathrm{~g} x_{n}\right), \varphi\left(\mathrm{g} x_{n-1}\right), \varphi\left(\mathrm{g} x_{n}\right)\right),\right. \\
& \left.\quad F\left(\sigma\left(\mathrm{g} y_{n-1}, \mathrm{~g} y_{n}\right), \varphi\left(\mathrm{g} y_{n-1}\right), \varphi\left(\mathrm{g} y_{n}\right)\right)\right\},
\end{aligned}
$$


which implies that $\left\{\max \left\{F\left(\sigma\left(\mathrm{g} x_{n}, \mathrm{~g} x_{n+1}\right), \varphi\left(\mathrm{g} x_{n}\right), \varphi\left(\mathrm{g} x_{n+1}\right)\right)\right.\right.$, $\left.\left.F\left(\sigma\left(\mathrm{g} y_{n}, \mathrm{~g} y_{n+1}\right), \varphi\left(\mathrm{g} y_{n}\right), \varphi\left(\mathrm{g} y_{n+1}\right)\right)\right\}\right\}$ is a decreasing nonnegative sequence. Therefore, there exists $r \geq 0$, such that

$$
\begin{gathered}
\lim _{n \longrightarrow \infty} \max \left\{F\left(\sigma\left(\mathrm{g} x_{n}, \mathrm{~g} x_{n+1}\right), \varphi\left(\mathrm{g} x_{n}\right), \varphi\left(\mathrm{g} x_{n+1}\right)\right),\right. \\
\left.F\left(\sigma\left(\mathrm{g} y_{n}, \mathrm{~g} y_{n+1}\right), \varphi\left(\mathrm{g} y_{n}\right), \varphi\left(\mathrm{g} y_{n+1}\right)\right)\right\}=r .
\end{gathered}
$$

Using condition (ii) of Definition 3, we get $r=0$. Then

$$
\begin{aligned}
& \lim _{n \longrightarrow \infty} \max \left\{\sigma\left(\mathrm{g} x_{n}, \mathrm{~g} x_{n+1}\right), \sigma\left(\mathrm{g} y_{n}, \mathrm{~g} y_{n+1}\right), \varphi\left(\mathrm{g} x_{n}\right),\right. \\
& \left.\varphi\left(\mathrm{g} y_{n}\right)\right\} \leq \lim _{n \longrightarrow \infty} \max \left\{F \left(\sigma\left(\mathrm{g} x_{n}, \mathrm{~g} x_{n+1}\right), \varphi\left(\mathrm{g} x_{n}\right),\right.\right. \\
& \left.\left.\varphi\left(\mathrm{g} x_{n+1}\right)\right), F\left(\sigma\left(\mathrm{g} y_{n}, \mathrm{~g} y_{n+1}\right), \varphi\left(\mathrm{g} y_{n}\right), \varphi\left(\mathrm{g} y_{n+1}\right)\right)\right\} \\
& =0 .
\end{aligned}
$$

Thus

$$
\begin{aligned}
\lim _{n \longrightarrow \infty} \sigma\left(\mathrm{g} x_{n}, \mathrm{~g} x_{n+1}\right) & =\lim _{n \longrightarrow+\infty} \sigma\left(\mathrm{g} y_{n}, \mathrm{~g} y_{n+1}\right) \\
& =\lim _{n \longrightarrow \infty} \varphi\left(\mathrm{g} x_{n}\right)=\lim _{n \longrightarrow \infty} \varphi\left(\mathrm{g} y_{n}\right) \\
& =0 .
\end{aligned}
$$

Next, we show that $\lim _{n, m \rightarrow \infty} \sigma\left(\mathrm{g} x_{n}, \mathrm{~g} x_{m}\right)=0$ and $\lim _{n, m \rightarrow \infty} \sigma\left(\mathrm{g} y_{n}, \mathrm{~g} y_{m}\right)=0$. Assume that this is not true; that is,

$$
\begin{array}{r}
\lim _{n, m \longrightarrow \infty} \sigma\left(\mathrm{g} x_{n}, \mathrm{~g} x_{m}\right) \neq 0 \\
\text { or } \lim _{n, m \longrightarrow \infty} \sigma\left(\mathrm{g} y_{n}, \mathrm{~g} y_{m}\right) \neq 0 .
\end{array}
$$

Then there exist $\epsilon>0, k_{1} \in \mathbb{N}^{+}$, and two subsequences $\left\{\mathrm{g} x_{m(k)}\right\},\left\{\mathrm{g} x_{n(k)}\right\}$ of $\left\{\mathrm{g} x_{n}\right\}$ with $m(k)>n(k)>k_{1}$, such that

$$
\begin{array}{r}
\max \left\{\sigma\left(\mathrm{g} x_{n(k)}, \mathrm{g} x_{m(k)}\right), \sigma\left(\mathrm{g} y_{n(k)}, \mathrm{g} y_{m(k)}\right)\right\} \geq \epsilon, \\
\max \left\{\sigma\left(\mathrm{g} x_{n(k)}, \mathrm{g} x_{m(k)-1}\right), \sigma\left(\mathrm{g} y_{n(k)}, \mathrm{g} y_{m(k)-1}\right)\right\}<\epsilon .
\end{array}
$$

With the definitions of metric-like spaces, we obtain

$$
\begin{aligned}
\sigma\left(\mathrm{g} x_{n(k)}, \mathrm{g} x_{m(k)}\right) \leq & \sigma\left(\mathrm{g} x_{n(k)}, \mathrm{g} x_{m(k)-1}\right) \\
& +\sigma\left(\mathrm{g} x_{m(k)-1}, \mathrm{~g} x_{m(k)}\right) \\
< & \epsilon+\sigma\left(\mathrm{g} x_{m(k)-1}, \mathrm{~g} x_{m(k)}\right), \\
\sigma\left(\mathrm{g} y_{n(k)}, \mathrm{g} y_{m(k)}\right) \leq & \sigma\left(\mathrm{g} y_{n(k)}, \mathrm{g} y_{m(k)-1}\right) \\
& +\sigma\left(\mathrm{g} y_{m(k)-1}, \mathrm{~g} y_{m(k)}\right) \\
< & \epsilon+\sigma\left(\mathrm{g} y_{m(k)-1}, \mathrm{~g} y_{m(k)}\right),
\end{aligned}
$$

which yields that

$$
\begin{aligned}
& \lim _{k \rightarrow \infty} \sigma\left(g x_{n(k)}, g x_{m(k)}\right) \leq \lim _{k \rightarrow \infty} \sigma\left(g x_{n(k)}, g x_{m(k)-1}\right) \\
& \quad \leq \epsilon .
\end{aligned}
$$

Similarly, we have

$$
\begin{aligned}
& \lim _{k \rightarrow \infty} \sigma\left(\mathrm{g} y_{n(k)}, \mathrm{g} y_{m(k)}\right) \leq \lim _{k \rightarrow \infty} \sigma\left(\mathrm{g} y_{n(k)}, \mathrm{g} y_{m(k)-1}\right) \\
& \quad \leq \epsilon .
\end{aligned}
$$

Thus, using (19) and (21), we obtain

$$
\begin{aligned}
\epsilon \leq & \max \left\{\lim _{k \rightarrow \infty} \sigma\left(\mathrm{g} x_{n(k)}, \mathrm{g} x_{m(k)}\right),\right. \\
& \left.\lim _{k \rightarrow \infty} \sigma\left(\mathrm{g} y_{n(k)}, \mathrm{g} y_{m(k)}\right)\right\} \\
& \leq \max \left\{\lim _{k \rightarrow \infty} \sigma\left(\mathrm{g} x_{n(k)}, \mathrm{g} x_{m(k)-1}\right),\right. \\
& \left.\lim _{k \longrightarrow \infty} \sigma\left(\mathrm{g} y_{n(k)}, \mathrm{g} y_{m(k)-1}\right)\right\} \leq \epsilon .
\end{aligned}
$$

Since

$$
\begin{aligned}
\sigma\left(\mathrm{g} x_{n(k)}, \mathrm{g} x_{m(k)}\right) \leq & \sigma\left(\mathrm{g} x_{n(k)}, \mathrm{g} x_{n(k)-1}\right) \\
& +\sigma\left(\mathrm{g} x_{n(k)-1}, \mathrm{~g} x_{m(k)-1}\right) \\
& +\sigma\left(\mathrm{g} x_{m(k)-1}, \mathrm{~g} x_{m(k)}\right) \\
\leq & \sigma\left(\mathrm{g} x_{n(k)}, \mathrm{g} x_{n(k)-1}\right) \\
& +\sigma\left(\mathrm{g} x_{n(k)-1}, \mathrm{~g} x_{n(k)}\right) \\
& +\sigma\left(\mathrm{g} x_{n(k)}, \mathrm{g} x_{m(k)-1}\right) \\
& +\sigma\left(\mathrm{g} x_{m(k)-1}, \mathrm{~g} x_{m(k)}\right) \\
< & \sigma\left(\mathrm{g} x_{n(k)}, \mathrm{g} x_{n(k)-1}\right) \\
& +\sigma\left(\mathrm{g} x_{n(k)-1}, \mathrm{~g} x_{n(k)}\right)+\epsilon \\
& +\sigma\left(\mathrm{g} x_{m(k)-1}, \mathrm{~g} x_{m(k)}\right),
\end{aligned}
$$

taking the limit as $k \longrightarrow+\infty$, we get

$$
\begin{aligned}
& \lim _{k \rightarrow \infty} \sigma\left(g x_{n(k)}, g x_{m(k)}\right) \leq \lim _{k \rightarrow \infty} \sigma\left(g x_{n(k)-1}, g x_{m(k)-1}\right) \\
& \quad \leq \epsilon .
\end{aligned}
$$

Similarly, we have

$$
\begin{aligned}
& \lim _{k \longrightarrow \infty} \sigma\left(\mathrm{g} y_{n(k)}, \mathrm{g} y_{m(k)}\right) \leq \lim _{k \longrightarrow \infty} \sigma\left(\mathrm{g} y_{n(k)-1}, \mathrm{~g} y_{m(k)-1}\right) \\
& \quad \leq \epsilon .
\end{aligned}
$$

Then it follows from the above inequalities that

$$
\begin{aligned}
\epsilon \leq & \max \left\{\lim _{k \rightarrow \infty} \sigma\left(\mathrm{g} x_{n(k)}, \mathrm{g} x_{m(k)}\right),\right. \\
& \left.\lim _{k \rightarrow \infty} \sigma\left(\mathrm{g} y_{n(k)}, \mathrm{g} y_{m(k)}\right)\right\} \\
& \leq \max \left\{\lim _{k \longrightarrow \infty} \sigma\left(\mathrm{g} x_{n(k)-1}, \mathrm{~g} x_{m(k)-1}\right),\right. \\
& \left.\lim _{k \rightarrow \infty} \sigma\left(\mathrm{g} y_{n(k)-1}, \mathrm{~g} y_{m(k)-1}\right)\right\} \leq \epsilon .
\end{aligned}
$$

Now, using (4), we have

$$
\begin{aligned}
\psi & \left(F\left(\sigma\left(\mathrm{g} x_{n(k)}, \mathrm{g} x_{m(k)}\right), \varphi\left(\mathrm{g} x_{n(k)}\right), \varphi\left(\mathrm{g} x_{m(k)}\right)\right)\right) \\
& =\psi\left(F \left(\sigma \left(T\left(x_{n(k)-1}, y_{n(k)-1}\right),\right.\right.\right.
\end{aligned}
$$




$$
\begin{aligned}
& \left.T\left(x_{m(k)-1}, y_{m(k)-1}\right)\right), \varphi\left(T\left(x_{n(k)-1}, y_{n(k)-1}\right)\right) \\
& \left.\left.\varphi\left(T\left(x_{m(k)-1}, y_{m(k)-1}\right)\right)\right)\right) \leq \phi\left(M \left(x_{n(k)-1}, x_{m(k)-1},\right.\right. \\
& \left.\left.y_{n(k)-1}, y_{m(k)-1}\right)\right)
\end{aligned}
$$

where

$$
\begin{aligned}
M & \left(x_{n(k)-1}, x_{m(k)-1}, y_{n(k)-1}, y_{m(k)-1}\right) \\
& =\max \left\{F \left(\sigma\left(\mathrm{g} x_{n(k)-1}, \mathrm{~g} x_{m(k)-1}\right), \varphi\left(\mathrm{g} x_{n(k)-1}\right),\right.\right. \\
& \left.\varphi\left(\mathrm{g} x_{m(k)-1}\right)\right), F\left(\sigma\left(\mathrm{g} y_{n(k)-1}, \mathrm{~g} y_{m(k)-1}\right), \varphi\left(\mathrm{g} y_{n(k)-1}\right),\right. \\
& \left.\varphi\left(\mathrm{g} y_{m(k)-1}\right)\right), F\left(\sigma\left(\mathrm{g} x_{n(k)-1}, T\left(x_{n(k)-1}, y_{n(k)-1}\right)\right),\right. \\
& \left.\varphi\left(\mathrm{g} x_{n(k)-1}\right), \varphi\left(T\left(x_{n(k)-1}, y_{n(k)-1}\right)\right)\right), \\
& F\left(\sigma\left(\mathrm{g} y_{n(k)-1}, T\left(y_{n(k)-1}, x_{n(k)-1}\right)\right), \varphi\left(\mathrm{g} y_{n(k)-1}\right),\right. \\
& \left.\left.\varphi\left(T\left(y_{n(k)-1}, x_{n(k)-1}\right)\right)\right)\right\} .
\end{aligned}
$$

Since

$$
\begin{aligned}
& \lim _{k \rightarrow \infty} \max \left\{F \left(\sigma\left(\mathrm{g} x_{n(k)-1}, T\left(x_{n(k)-1}, y_{n(k)-1}\right)\right),\right.\right. \\
& \left.\varphi\left(\mathrm{g} x_{n(k)-1}\right), \varphi\left(T\left(x_{n(k)-1}, y_{n(k)-1}\right)\right)\right), \\
& \quad F\left(\sigma\left(\mathrm{g} y_{n(k)-1}, T\left(y_{n(k)-1}, x_{n(k)-1}\right)\right), \varphi\left(\mathrm{g} y_{n(k)-1}\right),\right. \\
& \left.\left.\varphi\left(T\left(y_{n(k)-1}, x_{n(k)-1}\right)\right)\right)\right\}=0,
\end{aligned}
$$

there exists $k_{2} \in \mathbb{N}^{+}$with $k_{2}>k_{1}$, such that

$$
\begin{aligned}
& \max \left\{F \left(\sigma\left(\mathrm{g} x_{n(k)-1}, \mathrm{~g} x_{m(k)-1}\right), \varphi\left(\mathrm{g} x_{n(k)-1}\right),\right.\right. \\
& \left.\varphi\left(\mathrm{g} x_{m(k)-1}\right)\right), F\left(\sigma\left(\mathrm{g} y_{n(k)-1}, \mathrm{~g} y_{m(k)-1}\right), \varphi\left(\mathrm{g} y_{n(k)-1}\right),\right. \\
& \left.\varphi\left(\mathrm{g} y_{m(k)-1}\right)\right) \\
& \geq \max \left\{F \left(\sigma\left(\mathrm{g} x_{n(k)-1}, T\left(x_{n(k)-1}, y_{n(k)-1}\right)\right),\right.\right. \\
& \left.\varphi\left(\mathrm{g} x_{n(k)-1}\right), \varphi\left(T\left(x_{n(k)-1}, y_{n(k)-1}\right)\right)\right), \\
& F\left(\sigma\left(\mathrm{g} y_{n(k)-1}, T\left(\mathrm{y}_{n(k)-1}, x_{n(k)-1}\right)\right), \varphi\left(\mathrm{g} y_{n(k)-1}\right),\right. \\
& \left.\left.\varphi\left(T\left(y_{n(k)-1}, x_{n(k)-1}\right)\right)\right)\right\}
\end{aligned}
$$

for all $m(k)>n(k)>k_{2}$. Hence, we get

$$
\begin{aligned}
M & \left(x_{n(k)-1}, x_{m(k)-1}, y_{n(k)-1}, y_{m(k)-1}\right) \\
& =\max \left\{F \left(\sigma\left(\mathrm{g} x_{n(k)-1}, \mathrm{~g} x_{m(k)-1}\right), \varphi\left(\mathrm{g} x_{n(k)-1}\right),\right.\right. \\
& \left.\varphi\left(\mathrm{g} x_{m(k)-1}\right)\right), F\left(\sigma\left(\mathrm{g} y_{n(k)-1}, \mathrm{~g} y_{m(k)-1}\right),\right. \\
& \left.\left.\varphi\left(\mathrm{g} y_{n(k)-1}\right), \varphi\left(\mathrm{g} y_{m(k)-1}\right)\right)\right\},
\end{aligned}
$$

for all $m(k)>n(k)>k_{2}$. Similarly, there exists $k_{3} \in \mathbb{N}^{+}$with $k_{3}>k_{1}$, such that

$$
\begin{aligned}
\psi & \left(F\left(\sigma\left(\mathrm{g} y_{n(k)}, \mathrm{g} y_{m(k)}\right), \varphi\left(\mathrm{g} y_{n(k)}\right), \varphi\left(\mathrm{g} y_{m(k)}\right)\right)\right) \\
& =\psi\left(F \left(\sigma \left(T\left(y_{n(k)-1}, x_{n(k)-1}\right),\right.\right.\right. \\
& \left.T\left(y_{m(k)-1}, x_{m(k)-1}\right)\right), \varphi\left(T\left(y_{n(k)-1}, x_{n(k)-1}\right)\right), \\
& \left.\left.\varphi\left(T\left(y_{m(k)-1}, x_{m(k)-1}\right)\right)\right)\right) \leq \phi\left(M \left(y_{n(k)-1}, y_{m(k)-1},\right.\right. \\
& \left.\left.x_{n(k)-1}, x_{m(k)-1}\right)\right) \\
& =\phi\left(\operatorname { m a x } \left\{F \left(\sigma\left(\mathrm{g} x_{n(k)-1}, \mathrm{~g} x_{m(k)-1}\right), \varphi\left(\mathrm{g} x_{n(k)-1}\right),\right.\right.\right. \\
& \left.\varphi\left(\mathrm{g} x_{m(k)-1}\right)\right), F\left(\sigma\left(\mathrm{g} y_{n(k)-1}, \mathrm{~g} y_{m(k)-1}\right), \varphi\left(\mathrm{g} y_{n(k)-1}\right),\right. \\
& \left.\left.\left.\varphi\left(\mathrm{g} y_{m(k)-1}\right)\right)\right\}\right),
\end{aligned}
$$

for all $m(k)>n(k)>k_{3}$. Letting $k^{\prime}=\max \left\{k_{2}, k_{3}\right\}$ and noting that $\psi$ is nondecreasing, we obtain

$$
\begin{aligned}
& \psi\left(\operatorname { m a x } \left\{F\left(\sigma\left(\mathrm{g} x_{n(k)}, \mathrm{g} x_{m(k)}\right), \varphi\left(\mathrm{g} x_{n(k)}\right), \varphi\left(\mathrm{g} x_{m(k)}\right)\right),\right.\right. \\
& \left.\left.F\left(\sigma\left(\mathrm{g} y_{n(k)}, \mathrm{g} y_{m(k)}\right), \varphi\left(\mathrm{g} y_{n(k)}\right), \varphi\left(\mathrm{g} y_{m(k)}\right)\right)\right\}\right) \\
& =\max \left\{\psi \left(F \left(\sigma\left(\mathrm{g} x_{n(k)}, \mathrm{g} x_{m(k)}\right), \varphi\left(\mathrm{g} x_{n(k)}\right),\right.\right.\right. \\
& \left.\left.\varphi\left(\mathrm{g} x_{m(k)}\right)\right)\right), \psi\left(F \left(\sigma\left(\mathrm{g} y_{n(k)}, \mathrm{g} y_{m(k)}\right), \varphi\left(\mathrm{g} y_{n(k)}\right),\right.\right. \\
& \left.\left.\left.\varphi\left(\mathrm{g} y_{m(k)}\right)\right)\right)\right\} \leq \phi\left(\operatorname { m a x } \left\{F \left(\sigma\left(\mathrm{g} x_{n(k)-1}, \mathrm{~g} x_{m(k)-1}\right),\right.\right.\right. \\
& \left.\varphi\left(\mathrm{g} x_{n(k)-1}\right), \varphi\left(\mathrm{g} x_{m(k)-1}\right)\right), \\
& F\left(\sigma\left(\mathrm{g} y_{n(k)-1}, \mathrm{~g} y_{m(k)-1}\right), \varphi\left(\mathrm{g} y_{n(k)-1}\right),\right. \\
& \left.\left.\left.\varphi\left(\mathrm{g} y_{m(k)-1}\right)\right)\right\}\right),
\end{aligned}
$$

for all $m(k)>n(k)>k^{\prime}$. Taking the limit as $k \longrightarrow+\infty$ and using (25) and (29), we have

$$
\begin{aligned}
& \lim _{k \rightarrow \infty} \max \left\{F \left(\sigma\left(\mathrm{g} x_{n(k)}, \mathrm{g} x_{m(k)}\right), \varphi\left(\mathrm{g} x_{n(k)}\right),\right.\right. \\
& \left.\varphi\left(\mathrm{g} x_{m(k)}\right)\right), F\left(\sigma\left(\mathrm{g} y_{n(k)}, \mathrm{g} y_{m(k)}\right), \varphi\left(\mathrm{g} y_{n(k)}\right),\right. \\
& \left.\left.\varphi\left(\mathrm{g} y_{m(k)}\right)\right)\right\}=\lim _{k \rightarrow \infty} \max \left\{\sigma\left(\mathrm{g} x_{n(k)}, \mathrm{g} x_{m(k)}\right),\right. \\
& \left.\sigma\left(\mathrm{g} y_{n(k)}, \mathrm{g} y_{m(k)}\right)\right\}=\epsilon . \\
& \lim _{k \rightarrow \infty} \max \left\{F \left(\sigma\left(\mathrm{g} x_{n(k)-1}, \mathrm{~g} x_{m(k)-1}\right), \varphi\left(\mathrm{g} x_{n(k)-1}\right),\right.\right. \\
& \left.\varphi\left(\mathrm{g} x_{m(k)-1}\right)\right), F\left(\sigma\left(\mathrm{g} y_{n(k)-1}, \mathrm{~g} y_{m(k)-1}\right), \varphi\left(\mathrm{g} y_{n(k)-1}\right),\right. \\
& \left.\left.\varphi\left(\mathrm{g} y_{m(k)-1}\right)\right)\right\}=\lim _{k \rightarrow \infty} \max \left\{\sigma\left(\mathrm{g} x_{n(k)-1}, \mathrm{~g} x_{m(k)-1}\right),\right. \\
& \left.\sigma\left(\mathrm{g} y_{n(k)-1}, \mathrm{~g} y_{m(k)-1}\right)\right\}=\epsilon .
\end{aligned}
$$

According to the properties of $\Psi$, we get $\epsilon=0$, which is a contradiction. So, $\left\{\mathrm{g} x_{n}\right\}$ and $\left\{\mathrm{g} y_{n}\right\}$ are $\sigma$-Cauchy sequences in $(X, \sigma)$, which is a complete metric-like space. Since $\mathrm{T}(X \times$ $X) \subseteq \mathrm{g}(X)$ and $\mathrm{g}(X)$ is closed, there exist $x, y \in X$, such that $\lim _{n \rightarrow \infty} \mathrm{g} x_{n}=\mathrm{g} x, \lim _{n \rightarrow \infty} \mathrm{g} y_{n}=\mathrm{g} y$. Correspondingly, we have

$$
\begin{aligned}
& \sigma(\mathrm{g} x, \mathrm{~g} x)=\lim _{n \longrightarrow \infty} \sigma\left(\mathrm{g} x_{n}, \mathrm{~g} x\right)=0, \\
& \sigma(\mathrm{g} y, \mathrm{~g} y)=\lim _{n \longrightarrow \infty} \sigma\left(\mathrm{g} y_{n}, \mathrm{~g} y\right)=0 .
\end{aligned}
$$


Since $\varphi$ is a lower semicontinuous function, then $0 \leq \varphi(g x) \leq$ $\lim \inf _{n \rightarrow \infty} \varphi\left(g x_{n}\right)=0$. We have $\varphi(g x)=0$. By same arguments, we can derive that $\varphi(\mathrm{g} y)=0$.

Finally, we claim that $T$ and $g$ have a coupled coincidence point. It follows from (4) that

$$
\begin{aligned}
\psi & F\left(\sigma\left(T\left(x_{n}, y_{n}\right), T(x, y)\right), \varphi\left(T\left(x_{n}, y_{n}\right)\right),\right. \\
& \varphi(T(x, y)))) \leq \phi\left(M\left(x_{n}, x, y_{n}, y\right)\right) \\
& =\phi\left(\operatorname { m a x } \left\{F\left(\sigma\left(g x_{n}, \mathrm{~g} x\right), \varphi\left(\mathrm{g} x_{n}\right), \varphi(\mathrm{g} x)\right),\right.\right. \\
& F\left(\sigma\left(\mathrm{g} y_{n}, \mathrm{~g} y\right), \varphi\left(\mathrm{g} y_{n}\right), \varphi(\mathrm{g} y)\right), \\
& F\left(\sigma\left(\mathrm{g} x_{n}, T\left(x_{n}, y_{n}\right)\right), \varphi\left(\mathrm{g} x_{n}\right), \varphi\left(T\left(x_{n}, y_{n}\right)\right)\right), \\
& \left.\left.F\left(\sigma\left(\mathrm{g} y_{n}, T\left(y_{n}, x_{n}\right)\right), \varphi\left(\mathrm{g} y_{n}\right), \varphi\left(T\left(y_{n}, x_{n}\right)\right)\right)\right\}\right) \\
& =\phi\left(\operatorname { m a x } \left\{F\left(\sigma\left(\mathrm{g} x_{n}, \mathrm{~g} x\right), \varphi\left(\mathrm{g} x_{n}\right), \varphi(\mathrm{g} x)\right),\right.\right. \\
& F\left(\sigma\left(\mathrm{g} y_{n}, \mathrm{~g} y\right), \varphi\left(\mathrm{g} y_{n}\right), \varphi(\mathrm{g} y)\right), \\
& F\left(\sigma\left(\mathrm{g} x_{n}, \mathrm{~g} x_{n+1}\right), \varphi\left(\mathrm{g} x_{n}\right), \varphi\left(\mathrm{g} x_{n+1}\right)\right), \\
& \left.\left.F\left(\sigma\left(\mathrm{g} y_{n}, \mathrm{~g} y_{n+1}\right), \varphi\left(\mathrm{g} y_{n}\right), \varphi\left(\mathrm{g} y_{n+1}\right)\right)\right\}\right)
\end{aligned}
$$

Similarly, we have

$$
\begin{aligned}
\psi & F\left(\sigma\left(T\left(y_{n}, x_{n}\right), T(y, x)\right), \varphi\left(T\left(y_{n}, x_{n}\right)\right),\right. \\
& \varphi(T(y, x)))) \leq \phi\left(M\left(y_{n}, y, x_{n}, x\right)\right) \\
& =\phi\left(\operatorname { m a x } \left\{F\left(\sigma\left(\mathrm{g} y_{n}, \mathrm{~g} y\right), \varphi\left(\mathrm{g} y_{n}\right), \varphi(\mathrm{g} y)\right),\right.\right. \\
& F\left(\sigma\left(\mathrm{g} x_{n}, \mathrm{~g} x\right), \varphi\left(\mathrm{g} x_{n}\right), \varphi(\mathrm{g} x)\right), \\
& F\left(\sigma\left(\mathrm{g} y_{n}, T\left(y_{n}, x_{n}\right)\right), \varphi\left(\mathrm{g} y_{n}\right), \varphi\left(T\left(y_{n}, x_{n}\right)\right)\right), \\
& \left.\left.F\left(\sigma\left(\mathrm{g} x_{n}, T\left(x_{n}, y_{n}\right)\right), \varphi\left(\mathrm{g} x_{n}\right), \varphi\left(T\left(x_{n}, y_{n}\right)\right)\right)\right\}\right) \\
& =\phi\left(\operatorname { m a x } \left\{F\left(\sigma\left(\mathrm{g} y_{n}, \mathrm{~g} y\right), \varphi\left(\mathrm{g} y_{n}\right), \varphi(\mathrm{g} y)\right),\right.\right. \\
& F\left(\sigma\left(\mathrm{g} x_{n}, \mathrm{~g} x\right), \varphi\left(\mathrm{g} x_{n}\right), \varphi(\mathrm{g} x)\right), \\
& F\left(\sigma\left(\mathrm{g} y_{n}, \mathrm{~g} y_{n+1}\right), \varphi\left(\mathrm{g} y_{n}\right), \varphi\left(\mathrm{g} y_{n+1}\right)\right), \\
& \left.\left.F\left(\sigma\left(\mathrm{g} x_{n}, \mathrm{~g} x_{n+1}\right), \varphi\left(\mathrm{g} x_{n}\right), \varphi\left(\mathrm{g} x_{n+1}\right)\right)\right\}\right)
\end{aligned}
$$

Letting $n \longrightarrow \infty$ and using (38), we obtain

$$
\begin{aligned}
\lim _{n \longrightarrow \infty} M\left(x_{n}, x, y_{n}, y\right) & \\
= & \lim _{n \longrightarrow \infty} \max \left\{F\left(\sigma\left(\mathrm{g} x_{n}, \mathrm{~g} x\right), \varphi\left(\mathrm{g} x_{n}\right), \varphi(\mathrm{g} x)\right),\right. \\
& F\left(\sigma\left(\mathrm{g} y_{n}, \mathrm{~g} y\right), \varphi\left(\mathrm{g} y_{n}\right), \varphi(\mathrm{g} y)\right), \\
& F\left(\sigma\left(\mathrm{g} x_{n}, \mathrm{~g} x_{n+1}\right), \varphi\left(\mathrm{g} x_{n}\right), \varphi\left(\mathrm{g} x_{n+1}\right)\right), \\
& \left.F\left(\sigma\left(\mathrm{g} y_{n}, \mathrm{~g} y_{n+1}\right), \varphi\left(\mathrm{g} y_{n}\right), \varphi\left(\mathrm{g} y_{n+1}\right)\right)\right\}=0, \\
\lim _{n \longrightarrow \infty} & M\left(y_{n}, y, x_{n}, x\right) \\
= & \lim _{n \longrightarrow \infty} \max \left\{F\left(\sigma\left(\mathrm{g} y_{n}, \mathrm{~g} y\right), \varphi\left(\mathrm{g} y_{n}\right), \varphi(\mathrm{g} y)\right),\right.
\end{aligned}
$$

$$
\begin{aligned}
& F\left(\sigma\left(\mathrm{g} x_{n}, \mathrm{~g} x\right), \varphi\left(\mathrm{g} x_{n}\right), \varphi(\mathrm{g} x)\right), \\
& F\left(\sigma\left(\mathrm{g}\left(y_{n}\right), \mathrm{g}\left(y_{n+1}\right)\right), \varphi\left(\mathrm{g}\left(y_{n}\right)\right), \varphi\left(\mathrm{g}\left(y_{n+1}\right)\right)\right), \\
& \left.F\left(\sigma\left(\mathrm{g}\left(x_{n}\right), \mathrm{g}\left(x_{n+1}\right)\right), \varphi\left(\mathrm{g}\left(x_{n}\right)\right), \varphi\left(\mathrm{g}\left(x_{n+1}\right)\right)\right)\right\} \\
& =0 .
\end{aligned}
$$

Therefore, we get

$$
\begin{aligned}
& \lim _{n \rightarrow \infty} F\left(\sigma\left(T\left(x_{n}, y_{n}\right), T(x, y)\right), \varphi\left(T\left(x_{n}, y_{n}\right)\right),\right. \\
& \varphi(T(x, y)))=0, \\
& \lim _{n \longrightarrow \infty} F\left(\sigma\left(T\left(y_{n}, x_{n}\right), T(y, x)\right), \varphi\left(T\left(y_{n}, x_{n}\right)\right),\right. \\
& \varphi(T(y, x)))=0 .
\end{aligned}
$$

We can take advantage of the properties of $F$ to get

$$
\begin{aligned}
\lim _{n \longrightarrow \infty} \sigma\left(T\left(x_{n}, y_{n}\right), T(x, y)\right) \\
\quad=\lim _{n \longrightarrow \infty} \sigma\left(T\left(y_{n}, x_{n}\right), T(y, x)\right) \\
\quad=\lim _{n \longrightarrow \infty} \varphi\left(T\left(x_{n}, y_{n}\right)\right)=\lim _{n \longrightarrow \infty} \varphi\left(T\left(y_{n}, x_{n}\right)\right)=0 .
\end{aligned}
$$

By triangle inequality in metric-like space, we have

$$
\begin{aligned}
\sigma(\mathrm{g} x, T(x, y)) \leq & \sigma\left(\mathrm{g} x, \mathrm{~g} x_{n+1}\right)+\sigma\left(\mathrm{g} x_{n+1}, T(x, y)\right) \\
= & \sigma\left(\mathrm{g} x, \mathrm{~g} x_{n+1}\right) \\
& +\sigma\left(T\left(x_{n}, y_{n}\right), T(x, y)\right) .
\end{aligned}
$$

Letting $n \longrightarrow+\infty$, we get $\sigma(\mathrm{g} x, T(x, y))=0$. Similarly, we have $\sigma(\mathrm{g} y, T(y, x))=0$, that is, $\mathrm{g} x=T(x, y), \mathrm{g} y=T(y, x)$. Therefore, $T$ and $g$ have a coupled coincidence point.

Theorem 11. Define the partial order in $(X \times X, \preceq)$ by $(x, y) \preceq$ $\left(x^{*}, y^{*}\right) \Longleftrightarrow x \preceq x^{*}, y^{*} \preceq y$. Add to the hypotheses of Theorem 10 the following conditions:

(i) $\mathrm{T}$ and $\mathrm{g}$ commute at their coincidence points.

(ii) For every $(x, y)$ and $\left(x^{*}, y^{*}\right)$ in $X \times X$, there exists $(u, v) \in X \times X$ such that $(\mathrm{T}(u, v), \mathrm{T}(v, u))$ is comparable to $\left(\mathrm{T}\left(x^{*}, y^{*}\right), \mathrm{T}\left(y^{*}, x^{*}\right)\right)$ and $(\mathrm{T}(x, y), \mathrm{T}(y, x))$.

Then $\mathrm{T}$ and $\mathrm{g}$ have a unique common coupled fixed point.

Proof. First, by Theorem 10, we know that the set of coupled coincidence points is nonempty. Let $(x, y)$ and $\left(x^{*}, y^{*}\right)$ be coupled coincidence points, i.e., $\mathrm{g} x=T(x, y), \mathrm{g} y=T(y, x)$ and $\mathrm{g} x^{*}=T\left(x^{*}, y^{*}\right), \mathrm{g} y^{*}=T\left(y^{*}, x^{*}\right)$. Then we need to verify

$$
\begin{aligned}
& \mathrm{g} x=\mathrm{g} x^{*}, \\
& \mathrm{~g} y=\mathrm{g} y^{*}
\end{aligned}
$$

From (ii), there exists $(u, v) \in X \times X$ such that $(\mathrm{T}(u, v), \mathrm{T}(v, u))$ is comparable to $\left(\mathrm{T}\left(x^{*}, y^{*}\right), \mathrm{T}\left(y^{*}, x^{*}\right)\right)$, and $(\mathrm{T}(x, y), \mathrm{T}(y, x))$. Letting $u_{0}=u, \mathrm{v}_{0}=v$, we can choose 
$u_{1}, v_{1} \in X$ such that $\mathrm{g} u_{1}=T\left(u_{0}, v_{0}\right), \mathrm{g} v_{1}=T\left(v_{0}, u_{0}\right)$. Using this method, we can choose $u_{2}, v_{2} \in X$ such that $\mathrm{g} u_{2}=T\left(u_{1}, v_{1}\right), \mathrm{g} v_{2}=T\left(v_{1}, u_{1}\right)$. Repeating the procedures, we have $\left\{\mathrm{g} u_{n}\right\}$ and $\left\{\mathrm{g} v_{n}\right\}$ such that $\mathrm{g} u_{n}=T\left(u_{n-1}, v_{n-1}\right), \mathrm{g} v_{n}=$ $T\left(v_{n-1}, u_{n-1}\right)$. Similarly, letting $x_{0}=x, y_{0}=y, x_{0}^{*}=$ $x^{*}, y_{0}^{*}=y^{*}$, we get $\left\{\mathrm{g} x_{n}\right\},\left\{\mathrm{g} y_{n}\right\}$ and $\left\{\mathrm{g} x_{n}^{*}\right\},\left\{\mathrm{g} y_{n}^{*}\right\}$. Since $\mathrm{g} x=T(x, y), \mathrm{g} y=T(y, x)$, we get $\left(\mathrm{g} x_{0}, \mathrm{~g} y_{0}\right)=(\mathrm{g} x, \mathrm{~g} y)=$ $\left(T\left(x_{0}, y_{0}\right), T\left(y_{0}, x_{0}\right)\right)=\left(\mathrm{g} x_{1}, \mathrm{~g} y_{1}\right)$. And we have $\left(\mathrm{g} u_{1}, \mathrm{~g} v_{1}\right)=$ $(T(u, v), T(v, u))$, so $(\mathrm{g} x, \mathrm{~g} y) \preceq\left(\mathrm{g} u_{1}, \mathrm{~g} v_{1}\right)$, that is, $\mathrm{g} x \leq$ $\mathrm{g} u_{1}, \mathrm{~g} v_{1} \preceq \mathrm{g} y$. By condition (iii) of Theorem 10, we have $\mathrm{g} x \leq \mathrm{g} u_{n}, \mathrm{~g} v_{n} \leq \mathrm{g} y$. Using (4), we obtain

$$
\begin{gathered}
\psi\left(F\left(\sigma\left(\mathrm{g} x, \mathrm{~g} u_{n+1}\right), \varphi(\mathrm{g} x), \varphi\left(\mathrm{g} u_{n+1}\right)\right)\right) \\
=\psi\left(F \left(\sigma\left(T(x, y), T\left(u_{n}, v_{n}\right)\right), \varphi(T(x, y)),\right.\right. \\
\left.\left.\varphi\left(T\left(u_{n}, v_{n}\right)\right)\right)\right) \leq \phi\left(M\left(x, u_{n}, y, v_{n}\right)\right),
\end{gathered}
$$

where

$$
\begin{aligned}
& M\left(x, u_{n}, y, v_{n}\right) \\
& =\max \left\{F\left(\sigma\left(g x, g u_{n}\right), \varphi(g x), \varphi\left(g u_{n}\right)\right),\right. \\
& F\left(\sigma\left(\mathrm{g} y, \mathrm{~g} v_{n}\right), \varphi(\mathrm{g} y), \varphi\left(\mathrm{g} v_{n}\right)\right), \\
& F(\sigma(\mathrm{g} x, T(x, y)), \varphi(\mathrm{g} x), \varphi(T(x, y))), \\
& F(\sigma(\mathrm{g} y, T(y, x)), \varphi(\mathrm{g} y), \varphi(T(y, x)))\} \\
& =\max \left\{F\left(\sigma\left(\mathrm{g} x, \mathrm{~g} u_{n}\right), \varphi(\mathrm{g} x), \varphi\left(\mathrm{g} u_{n}\right)\right),\right. \\
& \left.F\left(\sigma\left(\mathrm{g} y, \mathrm{~g} v_{n}\right), \varphi(\mathrm{g} y), \varphi\left(\mathrm{g} v_{n}\right)\right)\right\} .
\end{aligned}
$$

Similarly,

$$
\begin{aligned}
\psi & \left(F\left(\sigma\left(\mathrm{g} y, \mathrm{~g} v_{n+1}\right), \varphi(\mathrm{g} y), \varphi\left(\mathrm{g} v_{n+1}\right)\right)\right) \\
& =\psi\left(F \left(\sigma\left(T(y, x), T\left(v_{n}, u_{n}\right)\right), \varphi(T(y, x)),\right.\right. \\
& \left.\left.\varphi\left(T\left(v_{n}, u_{n}\right)\right)\right)\right) \leq \phi\left(M\left(y, v_{n}, x, u_{n}\right)\right) \\
& =\max \left\{F\left(\sigma\left(\mathrm{g} x, \mathrm{~g} u_{n}\right), \varphi(\mathrm{g} x), \varphi\left(\mathrm{g} u_{n}\right)\right),\right. \\
& \left.F\left(\sigma\left(\mathrm{g} y, \mathrm{~g} v_{n}\right), \varphi(\mathrm{g} y), \varphi\left(\mathrm{g} v_{n}\right)\right)\right\} .
\end{aligned}
$$

Noting that $\psi$ is nondecreasing, we have

$$
\begin{aligned}
\psi( & \max \left\{F\left(\sigma\left(\mathrm{g} x, \mathrm{~g} u_{n+1}\right), \varphi(\mathrm{g} x), \varphi\left(\mathrm{g} u_{n+1}\right)\right),\right. \\
& \left.\left.F\left(\sigma\left(\mathrm{g} y, \mathrm{~g} v_{n+1}\right), \varphi(\mathrm{g} y), \varphi\left(\mathrm{g} v_{n+1}\right)\right)\right\}\right) \\
& \leq \phi\left(\operatorname { m a x } \left\{F\left(\sigma\left(\mathrm{g} x, \mathrm{~g} u_{n}\right), \varphi(\mathrm{g} x), \varphi\left(\mathrm{g} u_{n}\right)\right),\right.\right. \\
& \left.\left.F\left(\sigma\left(\mathrm{g} y, \mathrm{~g} v_{n}\right), \varphi(\mathrm{g} y), \varphi\left(\mathrm{g} v_{n}\right)\right)\right\}\right),
\end{aligned}
$$

so $\left\{\max \left\{F\left(\sigma\left(\mathrm{g} x, \mathrm{~g} u_{n+1}\right), \varphi(\mathrm{g} x), \varphi\left(\mathrm{g} u_{n+1}\right)\right), F\left(\sigma\left(\mathrm{g} y, \mathrm{~g} v_{n+1}\right)\right.\right.\right.$, $\left.\left.\left.\varphi(\mathrm{g} y), \varphi\left(\mathrm{g} v_{n+1}\right)\right)\right\}\right\}$ is a nonnegative decreasing sequence, and

$$
\begin{gathered}
\lim _{n \rightarrow \infty} \max \left\{F\left(\sigma\left(\mathrm{g} x, \mathrm{~g} u_{n+1}\right), \varphi(\mathrm{g} x), \varphi\left(\mathrm{g} u_{n+1}\right)\right),\right. \\
\left.F\left(\sigma\left(\mathrm{g} y, \mathrm{~g} v_{n+1}\right), \varphi(\mathrm{g} y), \varphi\left(\mathrm{g} v_{n+1}\right)\right)\right\}=0,
\end{gathered}
$$

which implies that

$$
\begin{aligned}
& \lim _{n \longrightarrow \infty} \sigma\left(\mathrm{g} x, \mathrm{~g} u_{n}\right)=\varphi(\mathrm{g} x)=0, \\
& \lim _{n \longrightarrow \infty} \sigma\left(\mathrm{g} y, \mathrm{~g} v_{n}\right)=\varphi(\mathrm{g} y)=0 .
\end{aligned}
$$

Through the same process, we can prove that

$$
\begin{aligned}
& \lim _{n \rightarrow \infty} \sigma\left(\mathrm{g} x^{*}, \mathrm{~g} u_{n}\right)=\varphi\left(\mathrm{g} x^{*}\right)=0, \\
& \lim _{n \longrightarrow \infty} \sigma\left(\mathrm{g} y^{*}, \mathrm{~g} v_{n}\right)=\varphi\left(\mathrm{g} y^{*}\right)=0 .
\end{aligned}
$$

Using triangle inequality, we have

$$
\begin{aligned}
& \sigma\left(\mathrm{g} x, \mathrm{~g} x^{*}\right) \leq \sigma\left(\mathrm{g} x, \mathrm{~g} u_{n}\right)+\sigma\left(\mathrm{g} x^{*}, \mathrm{~g} u_{n}\right) \\
& \sigma\left(\mathrm{g} y, \mathrm{~g} y^{*}\right) \leq \sigma\left(\mathrm{g} y, \mathrm{~g} v_{n}\right)+\sigma\left(\mathrm{g} y^{*}, \mathrm{~g} v_{n}\right)
\end{aligned}
$$

Letting $n \longrightarrow+\infty$, we get $\sigma\left(\mathrm{g} x, \mathrm{~g} x^{*}\right)=0, \sigma\left(\mathrm{g} y, \mathrm{~g} y^{*}\right)=0$, which implies that $\mathrm{g} x=\mathrm{g} x^{*}, \mathrm{~g} y=\mathrm{g} y^{*}$.

Denote $\mathrm{g} x=z, \mathrm{~g} y=w$. We now show that $(z, w)$ is a coupled common fixed point. Since $\mathrm{T}$ and $\mathrm{g}$ commute at their coincidence points, then we have

$$
\begin{gathered}
\mathrm{g} z=\mathrm{g}(\mathrm{g} x)=\mathrm{g}(T(x, y))=T(\mathrm{~g} x, \mathrm{~g} y)=T(z, w), \\
\mathrm{g} w=\mathrm{g}(\mathrm{g} y)=\mathrm{g}(T(y, x))=T(\mathrm{~g} y, \mathrm{~g} x)=T(w, z) .
\end{gathered}
$$

Hence, $(z, w)$ is a coupled coincidence point of T and g. Using (45), we have $\mathrm{g} z=\mathrm{g} x, \mathrm{~g} w=\mathrm{g} y$, and, therefore, $z=\mathrm{g} z=$ $T(z, w), w=\mathrm{g} w=T(w, z)$, which means $(z, w)$ is a coupled common fixed point.

Finally, we prove the existence and uniqueness of coupled common fixed point. If $(t, s)$ is also a coupled common fixed point, i.e., $t=\mathrm{g} t=T(t, s), s=\mathrm{g} s=T(s, t)$, then $(t, s)$ is also a coupled coincidence point. We have $t=\mathrm{g} t=\mathrm{g} z=z, s=\mathrm{g} s=$ $\mathrm{g} w=w$. Therefore, $\mathrm{T}$ and $\mathrm{g}$ have a unique common coupled fixed point.

Corollary 12. Define the partial order in $(X \times X, \preceq)$ by $(x, y) \preceq$ $\left(x^{*}, y^{*}\right) \Longleftrightarrow x \leq x^{*}, y^{*} \leq y$. Add to the hypotheses of Theorem 10 the following conditions:

(i) $\mathrm{T}$ and $\mathrm{g}$ commute at their coincidence points.

(ii) $\mathrm{g}(X)$ is a totally ordered subset of $X$.

Then $\mathrm{T}$ and $\mathrm{g}$ have a unique common coupled fixed point.

Proof. It is easy to see that condition (ii) of Theorem 11 can be naturally established.

Theorem 13. Under the hypotheses of Theorem 11, add the following condition: suppose that $\mathrm{g} y_{0} \leq \mathrm{g} x_{0}$. Then $\mathrm{T}$ and $\mathrm{g}$ have a unique common coupled fixed point of the form $(x, x)$.

Proof. Through the proof of Theorem 10, we construct the sequences $\left\{x_{n}\right\},\left\{y_{n}\right\} \in X$ such that $\mathrm{g} x_{n+1}=T\left(x_{n}, y_{n}\right), \mathrm{g} y_{n+1}=$ $T\left(y_{n}, x_{n}\right)$ and $\mathrm{g} x_{0} \preceq \mathrm{g} x_{1} \preceq \mathrm{g} x_{2} \preceq \cdots \preceq \mathrm{g} x_{n} \preceq \cdots$ and $\mathrm{g} y_{0} \geq \mathrm{g} y_{1} \geq \mathrm{g} y_{2} \geq \cdots \geq \mathrm{g} y_{n} \geq \cdots$. And $\mathrm{g}(X)$ is closed; then there exist $x, y \in X$, such that $\lim _{n \rightarrow \infty} \mathrm{g} x_{n}=$ $\mathrm{g} x, \lim _{n \rightarrow \infty} \mathrm{g} y_{n}=\mathrm{g} y$. In Theorem 11, we prove that $(x, y)$ is the unique coupled common fixed point of $\mathrm{T}$ and g, i.e., $x=\mathrm{g} x=T(x, y), y=\mathrm{g} y=T(y, x)$. 
Next, we shall show $\mathrm{g} x=T(x, y)=\mathrm{g} y=T(y, x)$. Owing to the supplementary condition, we have gy $\leq \mathrm{g} y_{n} \preceq \cdots \preceq$ $\mathrm{g} y_{0} \preceq \mathrm{g} x_{0} \preceq \mathrm{g} x_{1} \preceq \cdots \preceq \mathrm{g} x_{n} \preceq \mathrm{g} x$. From (4), we obtain

$$
\begin{aligned}
\psi & (F(\sigma(\mathrm{g} x, \mathrm{~g} y), \varphi(\mathrm{g} x), \varphi(\mathrm{g} y))) \leq \phi(M(x, y, y, x)) \\
& =\phi(\max \{F(\sigma(\mathrm{g} x, \mathrm{~g} y), \varphi(\mathrm{g} x), \varphi(\mathrm{g} y)), \\
& F(\sigma(\mathrm{g} y, \mathrm{~g} x), \varphi(\mathrm{g} y), \varphi(\mathrm{g} x))\}) .
\end{aligned}
$$

By (10), we get $\varphi(\mathrm{g} x)=\varphi(\mathrm{g} y)=0$, and thus we have

$$
\begin{gathered}
F(\sigma(\mathrm{g} x, \mathrm{~g} y), \varphi(\mathrm{g} x), \varphi(\mathrm{g} y))=\sigma(\mathrm{g} x, \mathrm{~g} y) \\
\quad=F(\sigma(\mathrm{g} y, \mathrm{~g} x), \varphi(\mathrm{g} y), \varphi(\mathrm{g} x)) .
\end{gathered}
$$

So (55) can be converted into

$$
\psi(\sigma(\mathrm{g} x, \mathrm{~g} y)) \leq \phi(\sigma(\mathrm{g} x, \mathrm{~g} y))
$$

which implies that $\mathrm{g} x=\mathrm{g} y$. Then $\mathrm{T}$ and $\mathrm{g}$ have a unique common coupled fixed point of the form $(x, x)$.

Example 14. Let $X=[0,+\infty)$ and $\sigma: X \times X \longrightarrow X$ be defined by

$$
\sigma(x, y)= \begin{cases}2 x, & \text { if } x=y, \\ \max \{x, y\}, & \text { otherwise. }\end{cases}
$$

Then $(X, \sigma)$ is a complete metric-like space, rather than a partial metric space. Indeed, if $x<y<2 x$, we obtain $\sigma(x, x)>\sigma(x, y)$. Let $T: X \times X \longrightarrow X$ be defined as

$$
T(x, y)= \begin{cases}\frac{x-y}{6}, & x \geq y, \\ 0, & x<y .\end{cases}
$$

Define function $g: X \longrightarrow X$ by $g x=x$. Let $\psi, \varphi: X \longrightarrow X$ be defined by

$$
\begin{aligned}
& \psi(t)=\ln (1+t), \\
& \phi(t)=\ln \left(1+\frac{t}{2}\right) .
\end{aligned}
$$

Let $\varphi: X \longrightarrow X$ be defined by $\varphi(t)=t$ and $F:[0, \infty)^{3} \longrightarrow$ $[0, \infty)$ be the function defined by $F(a, b, c)=a+b+c$.

It is clear that $T$ has the mixed g-monotone property. Easily, we can know $\mathrm{g}(X)$ is closed and $T(X \times X) \subseteq \mathrm{g}(X)$. Letting $x_{0}=y_{0}=0$, then $x_{0} \leq T\left(x_{0}, y_{0}\right), T\left(y_{0}, x_{0}\right) \leq y_{0}$ is true. And if $\psi(u)=\ln (1+u) \leq \phi(v)=\ln (1+v / 2)$, we have $u \leq v / 2$; i.e., $u \leq v$ is established, so $\psi, \varphi \in \Psi$. And $\mathrm{g} T(x, y)=T(\mathrm{~g} x, \mathrm{~g} y)$, and then $\mathrm{g}$ and $T$ are commutative. Next, we prove that (4) holds for all $x, y, u, v \in X$ with $x \leq u, v \leq y$. We can distinguish three cases.

Case $1(v \leq y \leq x \leq u)$. If $T(x, y)=T(u, v)$, we get $v=y$ and $x=u$.

$$
\begin{aligned}
\psi & (F(\sigma(T(x, y), T(u, v)), \varphi(T(x, y)), \varphi(T(u, v)))) \\
\quad & \ln \left[1+2 \cdot \frac{x-y}{6}+\frac{x-y}{6}+\frac{u-v}{6}\right]=\ln [1 \\
& \left.+\frac{x-y}{2}+\frac{u-v}{6}\right], \\
\phi & (M(x, u, y, v))=\ln \left[1+\frac{1}{2} \times \max \{4 x, 4 y, 2 x\right. \\
& \left.\left.+\frac{x-y}{6}, 2 y\right\}\right]=\ln [1+2 u] .
\end{aligned}
$$

If $T(x, y) \neq T(u, v)$, we get $v \neq y$ or $x \neq u$.

$$
\begin{aligned}
& \psi(F(\sigma(T(x, y), T(u, v)), \varphi(T(x, y)), \varphi(T(u, v))))=\ln [1+\max \{T(x, y), T(u, v)\}+T(x, y)+T(u, v)] \\
& \quad=\ln \left[1+\frac{u-v}{3}+\frac{x-y}{6}\right], \\
& M(x, u, y, v)= \begin{cases}\max \left\{4 u, 2 y+v, 2 x+\frac{x-y}{6}, 2 y\right\}, & \text { if } v \neq y \text { and } x=u, \\
\max \left\{2 u+x, 4 y, 2 x+\frac{x-y}{6}, 2 y\right\}, & \text { if } x \neq u \text { and } v=y, \\
\max \left\{2 u+x, 2 y+v, 2 x+\frac{x-y}{6}, 2 y\right\}, & \text { if } v \neq y \text { and } x \neq u .\end{cases}
\end{aligned}
$$

Then we have

$$
\phi(M(x, u, y, v)) \geq \ln \left[1+u+\frac{x}{2}\right] .
$$

Hence, $\psi(F(\sigma(T(x, y), T(u, v)), \varphi(T(x, y)), \varphi(T(u, v)))) \leq$ $\phi(M(x, u, y, v))$ is proved.
Case $2(x \leq u, x<y, v \leq y, u \geq v)$. If $T(x, y)=T(u, v)$, we have $T(x, y)=0$ and $u=v$.

$$
\begin{aligned}
& \psi(F(\sigma(T(x, y), T(u, v)), \varphi(T(x, y)), \varphi(T(u, v)))) \\
& \quad=0
\end{aligned}
$$


If $T(x, y) \neq T(u, v)$, we have $T(x, y)=0$ and $u \neq v$.

$$
\begin{aligned}
& \psi\left(F(\sigma(T(x, y), T(u, v)), \varphi(T(x, y)), \varphi(T(u, v)))=\ln \left[1+\frac{u-v}{3}\right],\right. \\
& M(x, u, y, v)= \begin{cases}\max \left\{4 u, 2 y+v, 2 x, 2 y+\frac{y-x}{6}\right\}, & \text { if } v \neq y \text { and } x=u, \\
\max \left\{4 u, 4 y, 2 x, 2 y+\frac{y-x}{6}\right\}, & \text { if } x=u \text { and } v=y, \\
\max \left\{2 u+x, 4 y, 2 x, 2 y+\frac{y-x}{6}\right\}, & \text { if } v=y \text { and } x \neq u, \\
\max \left\{2 u+x, 2 y+v, 2 x, 2 y+\frac{y-x}{6}\right\}, & \text { if } v \neq y \text { and } x \neq u .\end{cases}
\end{aligned}
$$

Then we have

$$
\phi(M(x, u, y, v)) \geq \ln \left[1+u+\frac{x}{2}\right] .
$$

Hence, $\psi(F(\sigma(T(x, y), T(u, v)), \varphi(T(x, y)), \varphi(T(u, v)))) \leq$ $\phi(M(x, u, y, v))$ is tenable.

Case $3(x \leq u, x<y, v \leq y, v<u)$.

$$
\begin{aligned}
& \psi(F(\sigma(T(x, y), T(u, v)), \varphi(T(x, y)), \varphi(T(u, v)))) \\
& \quad=0 \leq \phi(M(x, u, y, v)) .
\end{aligned}
$$

Hence, (4) is established. And (4) still holds with $u \leq x, y \leq v$.

Note that all conditions of Theorems 10-13 are satisfied. Hence, $T$ and $g$ have a unique common coupled fixed point, which is $(0,0)$.

\section{Consequences of the Main Result}

By choosing suitable mappings $\psi, \phi$, g, and $F$, one can deduce subsequent corollaries.

Letting $g=I$ (the identity mapping on $X$ ) in Theorem 10 , we get the following corollary.

Corollary 15. Let $(X, \preceq)$ be a partially ordered set and $(X, \sigma)$ be a complete metric-like space. Let $\mathrm{T}: X \times X \longrightarrow X$ be a mapping, satisfying the following conditions:

(i) Thas the mixed monotone property.

(ii) There exist $x_{0}, y_{0} \in X$ such that $x_{0} \leq T\left(x_{0}, y_{0}\right), T\left(y_{0}\right.$, $\left.x_{0}\right) \leq y_{0}$ and $y_{0} \leq x_{0}$.

(iii) $\psi(F(\sigma(T(x, y), T(u, v)), \varphi(T(x, y)), \varphi(T(u, v)))) \leq$ $\phi(M(x, u, y, v))$, where

$$
\begin{gathered}
M(x, u, y, v)=\max \{F(\sigma(x, u), \varphi(x), \varphi(u)), \\
F(\sigma(y, v), \varphi(y), \varphi(v)), \\
F(\sigma(x, T(x, y)), \varphi(x), \varphi(T(x, y))), \\
F(\sigma(y, T(y, x)), \varphi(y), \varphi(T(y, x)))\},
\end{gathered}
$$

with $x \leq u$ and $v \leq y$, or $u \leq x$ and $y \leq v$, for all $x, y, u, v \in$ $X, F \in \mathbb{F},(\psi, \phi) \in \Psi, \psi$ is nondecreasing, and $\varphi: X \longrightarrow$ $[0,+\infty)$ is a lower semicontinuous function.

Then $T$ has a coupled fixed point.
Setting $F(a, b, c)=a+b+c$ and $\varphi(t)=0, t \geq 0$ in Theorem 10, we get another corollary.

Corollary 16. Let $(X, \preceq)$ be a partially ordered set and $(X, \sigma)$ be a complete metric-like space. Let $\mathrm{T}: X \times X \longrightarrow X$ and $\mathrm{g}$ : $X \longrightarrow X$ be two mappings such that the following conditions are satisfied:

(i) $\mathrm{T}(X \times X) \subseteq \mathrm{g}(X)$.

(ii) $\mathrm{g}(X)$ is closed.

(iii) $T$ has the mixed g-monotone property.

(iv) There exist $x_{0}, y_{0} \in X$ such that $\mathrm{g} x_{0} \leq T\left(x_{0}, y_{0}\right)$, $T\left(y_{0}, x_{0}\right) \preceq \mathrm{g} y_{0}$.

(v)

$$
\begin{gathered}
\psi(\sigma(T(x, y), T(u, v))) \leq \phi(\max \{\sigma(\mathrm{g} x, \mathrm{~g} u), \\
\sigma(\mathrm{g} y, \mathrm{~g} v), \sigma(\mathrm{g} x, T(x, y)), \sigma(\mathrm{g} y, T(y, x))\}),
\end{gathered}
$$

with $x \preceq u$ and $v \preceq y$, or $u \preceq x$ and $y \preceq v$, for all $x, y, u, v \in$ $X,(\psi, \phi) \in \Psi$, and $\psi$ is nondecreasing.

Then $T$ and $g$ have a coupled coincidence point.

Corollary 17. Let $(X, \preceq)$ be a partially ordered set and $(X, \sigma)$ be a complete metric-like space. Let $\mathrm{T}: X \times X \longrightarrow X$ and $\mathrm{g}$ : $X \longrightarrow X$ be two mappings, such that the following conditions are satisfied:

(i) $\mathrm{T}(X \times X) \subseteq \mathrm{g}(X)$.

(ii) $\mathrm{g}(X)$ is closed.

(iii) $T$ has the mixed g-monotone property.

(iv) There exist $x_{0}, y_{0} \in X$ such that $\mathrm{g} x_{0} \leq T\left(x_{0}, y_{0}\right)$, $T\left(y_{0}, x_{0}\right) \preceq \mathrm{g} y_{0}$.

(v)

$$
\begin{aligned}
\sigma(T(x, y), T(u, v)) \leq & a \sigma(\mathrm{g} x, \mathrm{~g} u)+b \sigma(\mathrm{g} y, \mathrm{~g} v) \\
& +c \sigma(\mathrm{g} x, T(x, y)) \\
& +d \sigma(\mathrm{g} y, T(y, x))
\end{aligned}
$$

with $x \preceq u$ and $v \preceq y$, or $u \preceq x$ and $y \preceq v$, for all $x, y, u, v \in$ $X, a, b, c, d \in \mathbb{R}^{+}, a+b+c+d \in[0,1)$.

Then $T$ and $\mathrm{g}$ have a coupled coincidence point. 
Proof. Denote $\psi(t)=t, \phi(t)<t$, we can note that

$$
\begin{aligned}
& a \sigma(\mathrm{g} x, \mathrm{~g} u)+b \sigma(\mathrm{g} y, \mathrm{~g} v)+c \sigma(\mathrm{g} x, T(x, y)) \\
& \quad+d \sigma(\mathrm{g} y, T(y, x)) \leq(a+b+c+d) \\
& \quad \cdot \max \{\sigma(\mathrm{g} x, \mathrm{~g} u), \sigma(\mathrm{g} y, \mathrm{~g} v), \sigma(\mathrm{g} x, T(x, y)), \\
& \quad \sigma(\mathrm{g} y, T(y, x))\} .
\end{aligned}
$$

Hence, the conditions of Corollary 16 can be satisfied.

Since metric spaces are metric-like spaces, corresponding theorems in metric spaces can be derived. On the other hand, according to the definition of $\Psi$, if $(\psi, \phi) \in \Psi$, we obtain $\psi-$ $\phi \in \Psi$. Now we have the following result in metric spaces.

Theorem 18. Let $(X, \preceq)$ be a partially ordered set and $(X, d)$ be a complete metric space. Let $\mathrm{T}: X \times X \longrightarrow X$ and $\mathrm{g}$ : $X \longrightarrow X$ be two mappings such that the following conditions are satisfied:

(i) $\mathrm{T}(X \times X) \subseteq \mathrm{g}(X)$.

(ii) $\mathrm{g}(X)$ is closed.

(iii) $T$ has the mixed g-monotone property.

(iv) There exist $x_{0}, y_{0} \in X$ such that $\mathrm{g} x_{0} \preceq T\left(x_{0}, y_{0}\right)$ and $T\left(y_{0}, x_{0}\right) \preceq \mathrm{g} y_{0}$.

(v) $\psi(F(d(T(x, y), T(u, v)), \varphi(T(x, y)), \varphi(T(u, v)))) \leq$ $\psi(M(x, u, y, v))-\phi(M(x, u, y, v))$, where

$$
\begin{aligned}
& M(x, u, y, v)=\max \{F(d(\mathrm{~g} x, \mathrm{~g} u), \varphi(\mathrm{g} x), \varphi(\mathrm{g} u)), \\
& \quad F(d(\mathrm{~g} y, \mathrm{~g} v), \varphi(\mathrm{g} y), \varphi(\mathrm{g} v)) \\
& \quad F(d(\mathrm{~g} x, T(x, y)), \varphi(\mathrm{g} x), \varphi(T(x, y))) \\
& \quad F(d(\mathrm{~g} y, T(y, x)), \varphi(\mathrm{g} y), \varphi(T(y, x)))\}
\end{aligned}
$$

with $x \preceq u$ and $v \preceq y$, or $u \preceq x$ and $y \preceq v$, for all $x, y, u, v \in$ $X, F \in \mathbb{F},(\psi, \phi) \in \Psi, \psi$ is nondecreasing, and $\varphi: X \longrightarrow$ $[0,+\infty)$ is a lower semicontinuous function.

Then $T$ and $g$ have a coupled coincidence point.

\section{An Application}

In this section, we study the existence of a unique solution to a system of nonlinear integral equations by applying a coupled fixed point result in Section 3. Consider the following system of equations:

$$
\begin{gathered}
x(t)=h(t)+\int_{0}^{1} k_{1}(t, s) f_{1}(s, x(s)) d s \\
\cdot \int_{0}^{1} k_{2}(t, s) f_{2}(s, y(s)) d s \\
y(t)=h(t)+\int_{0}^{1} k_{1}(t, s) f_{1}(s, y(s)) d s \\
\cdot \int_{0}^{1} k_{2}(t, s) f_{2}(s, x(s)) d s
\end{gathered}
$$

Now, we discuss the existence of the unique solution.

Theorem 19. Let $X=C(I)$ be the set of all continuous functions defined on $I=[0,1]$ with a dislocated metric given by $\sigma(x, y)=\sup _{t \in I}|x(t)-y(t)|$. Assume that $T: X \times X \longrightarrow X$ has the mixed monotone property, and $T$ is defined by

$$
\begin{aligned}
& T(x, y)(t)=h(t)+\int_{0}^{1} k_{1}(t, s) f_{1}(s, x(s)) d s \\
& \cdot \int_{0}^{1} k_{2}(t, s) f_{2}(s, y(s)) d s \\
& T(y, x)(t)=h(t)+\int_{0}^{1} k_{1}(t, s) f_{1}(s, y(s)) d s \\
& \quad \cdot \int_{0}^{1} k_{2}(t, s) f_{2}(s, x(s)) d s
\end{aligned}
$$

which satisfy the following conditions:

(i) $h:[0,1] \longrightarrow \mathbb{R}$ is continuous.

(ii) $k_{i}:[0,1] \times[0,1] \longrightarrow \mathbb{R}^{+}$and $f_{i}:[0,1] \times \mathbb{R} \longrightarrow$ $\mathbb{R}^{+}(i=1,2)$ are continuous. There exists a constant $D_{i} \in \mathbb{R}^{+}$ such that $\int_{0}^{1} k_{i}(t, s) d s \leq D_{i}$. And $\int_{0}^{1} k_{i}(t, s) f_{i}(s, x(s)) d s \leq K$, where $K \geq 0$ is a constant real number.

(iii) $\phi \in \Psi$ is nondecreasing and $\phi(t)<t$. There exists $a$ constant $L_{i} \in[0,1)$ such that for all $x, y \in X$,

$$
\left|f_{i}(t, x)-f_{i}(t, y)\right| \leq L_{i} \phi(|x-y|) \quad(i=1,2) .
$$

(iv) $2 K \cdot \max \left\{L_{1} D_{1}, L_{2} D_{2}\right\}<1$.

If $x \preceq u, v \preceq y$, or $u \preceq x, y \preceq v$, and there exist $x_{0}, y_{0} \in X$ such that $x_{0} \preceq T\left(x_{0}, y_{0}\right)$ and $T\left(y_{0}, x_{0}\right) \preceq y_{0}$, then $T$ has a coupled fixed point; i.e., (83) has a unique solution in $C(I)$.

Proof. Define $\sigma(x, y)=\sup _{t \in I}|x(t)-y(t)|$. Letting $x \preceq u, v \preceq$ $y$, or $u \preceq x, y \preceq v$, and $x, y, u, v \in X$, we have

$$
\begin{aligned}
\mid T & (x, y)(t)-T(u, v)(t) \mid \\
& =\left|\int_{0}^{1} k_{1}(t, s) f_{1}(s, x(s)) d s \int_{0}^{1} k_{2}(t, s) f_{2}(s, y(s)) d s-\int_{0}^{1} k_{1}(t, s) f_{1}(s, u(s)) d s \int_{0}^{1} k_{2}(t, s) f_{2}(s, v(s)) d s\right| \\
& \leq\left|\int_{0}^{1} k_{1}(t, s) f_{1}(s, x(s)) d s \int_{0}^{1} k_{2}(t, s)\left[f_{2}(s, y(s))-f_{2}(s, v(s))\right] d s\right| \\
& +\left|\int_{0}^{1} k_{1}(t, s)\left[f_{1}(s, x(s))-f_{1}(s, u(s))\right] d s \int_{0}^{1} k_{2}(t, s) f_{2}(s, v(s)) d s\right| \leq K \cdot \int_{0}^{1} k_{2}(t, s) L_{2}(\phi(\sigma(y(s), v(s)))) d s+K \\
& \cdot \int_{0}^{1} k_{1}(t, s) L_{1}(\phi(\sigma(u(s), x(s)))) d s \leq K \cdot \int_{0}^{1} k_{2}(t, s) L_{2}(\phi(\sigma(y, v))) d s+K \cdot \int_{0}^{1} k_{1}(t, s) L_{1}(\phi(\sigma(u, x))) d s \leq 2 K \\
& \cdot \max \left\{L_{1} D_{1}, L_{2} D_{2}\right\} \cdot \phi(\max \{\sigma(y, v), \sigma(u, x)\}) \leq \phi(\max \{\sigma(y, v), \sigma(u, x), \sigma(T(x, y), u), \sigma(T(y, x), v)\})
\end{aligned}
$$


Hence, we get $\sigma(T(x, y), T(u, v)) \leq \phi(\max \{\sigma(y, v), \sigma(u$, $x), \sigma(T(x, y), u), \sigma(T(y, x), v)\})$. On the other hand, by the assumptions of the theorem, the other conditions of Corollary 16 are satisfied with $g t=t$. Then $T$ has a coupled fixed point; i.e., (73) has a unique solution in $C(I)$.

Example 20. Consider the following system of nonlinear integral equations:

$$
\begin{aligned}
x(t)= & \ln (t+1)+\int_{0}^{1} s e^{t} \cdot \frac{s^{2}}{3\left(1+s^{4}\right)} \\
& \cdot \frac{|x(s)|}{1+|x(s)|} d s \int_{0}^{1} \frac{s}{1+t^{2}} \\
& \cdot \frac{1}{3\left(1+s^{2}\right)} \frac{1+|y(s)|}{(2+|y(s)|)^{2}} d s \\
y(t)= & \ln (t+1)+\int_{0}^{1} s e^{t} \cdot \frac{s^{2}}{3\left(1+s^{4}\right)} \\
& \cdot \frac{|y(s)|}{1+|y(s)|} d s \int_{0}^{1} \frac{s}{1+t^{2}} \\
& \cdot \frac{1}{3\left(1+s^{2}\right)} \frac{1+|x(s)|}{(2+|x(s)|)^{2}} d s,
\end{aligned}
$$

where $t \in[0,1]$. Then we can define the functions by

$$
\begin{aligned}
h(t) & =\ln (t+1) \\
k_{1}(t, s) & =s e^{t}, \\
k_{2}(t, s) & =\frac{s}{1+t^{2}} \\
f_{1}(s, x(s)) & =\frac{s^{2}}{3\left(1+s^{4}\right)} \cdot \frac{|x(s)|}{1+|x(s)|} \\
f_{2}(s, x(s)) & =\frac{1}{3\left(1+s^{2}\right)} \cdot \frac{|y(s)|}{(2+|y(s)|)^{2}}
\end{aligned}
$$

It is clear that $h$ and $f_{i}, k_{i}(\mathrm{i}=1,2)$ are continuous functions, and $f_{i}(t, x) \geq 0$. Supposing $\phi(t)=(1 / 3) t$, we obtain $\phi \in \Psi$ with $\phi(t)<t$ and $\phi$ is nondecreasing. Since

$$
\begin{aligned}
& \left|f_{1}(t, x(t))-f_{1}(t, u(t))\right| \\
& \quad=\frac{t^{2}}{3\left(1+t^{4}\right)} \cdot\left|\frac{|x(t)|}{1+|x(t)|}-\frac{|u(t)|}{1+|u(t)|}\right| \\
& \quad \leq \frac{1}{6}|x-u|=\frac{1}{2} \phi(x-u), \\
& \left|f_{2}(t, y(t))-f_{2}(t, v(t))\right| \\
& \quad=\frac{1}{3\left(1+t^{2}\right)} \cdot\left|\frac{1+|y(t)|}{(2+|y(t)|)^{2}}-\frac{1+|v(t)|}{(2+|v(t)|)^{2}}\right| \\
& \quad \leq \frac{1}{6}|y-v|=\frac{1}{2} \phi(y-v),
\end{aligned}
$$

we can get $L_{1}=L_{2}=1 / 2$. And we have

$$
\begin{aligned}
& \int_{0}^{1} k_{1}(t, s) f_{1}(s, x(s)) d s \leq \int_{0}^{1} s e^{t} \cdot \frac{s^{2}}{3\left(1+s^{4}\right)} d s \\
& \quad=\frac{1}{12} \ln 2 \cdot e^{t} \leq \frac{1}{12} \ln 2 \cdot e, \\
& \int_{0}^{1} k_{2}(t, s) f_{2}(s, x(s)) d s \leq \int_{0}^{1} \frac{s}{1+t^{2}} \cdot \frac{1}{3\left(1+s^{2}\right)} d s \\
& \quad \leq \frac{1}{6} \ln 2 .
\end{aligned}
$$

So, letting $K=(1 / 12) \ln 2 \cdot e$, we have $\int_{0}^{1} k_{i}(t, s) f_{i}(s, x(s)) d s \leq$ K.

$$
\begin{aligned}
& \int_{0}^{1} k_{1}(t, s) d s \leq \frac{1}{2} e^{t} \leq \frac{1}{2} e=D_{1}, \\
& \int_{0}^{1} k_{2}(t, s) d s \leq \frac{1}{2\left(1+t^{2}\right)} \leq \frac{1}{4}=D_{2} .
\end{aligned}
$$

Through calculation, we can see $2 K \cdot \max \left\{L_{1} D_{1}, L_{2} D_{2}\right\}<1$. Hence, all conditions of Theorem 19 are satisfied; then the integral equations have a unique solution in $C(I)$, where $I=$ $[0,1]$.

Remark 21. Notice that the given $\sigma$, which is defined by $\sigma(x, y)=\sup _{t \in I}|x(t)-y(t)|$, is a metric. Next, we consider an application in a metric-like space. Let $X=C(I)$ be the set of all continuous functions with a dislocated metric given by

$$
\sigma(x, y)=\sup _{t \in I}(|x(t)|+|y(t)|), \quad \forall x, y \in X .
$$

Clearly, $(X, \sigma)$ is a complete metric-like space but not a metric space.

Theorem 22. Let $X=C(I)$ be the set of all continuous functions defined on $I=[0,1]$ with a dislocated metric given by $\sigma(x, y)=\sup _{t \in I}(|x(t)|+|y(t)|)$. Assume that $T: X \times X \longrightarrow X$ has the mixed monotone property, and $T$ is defined by

$$
\begin{aligned}
& T(x, y)(t) \\
& =\int_{0}^{1} k_{1}(t, s) f_{1}(s, x(s)) d s \int_{0}^{1} k_{2}(t, s) f_{2}(s, y(s)) d s \\
& T(y, x)(t) \\
& =\int_{0}^{1} k_{1}(t, s) f_{1}(s, y(s)) d s \int_{0}^{1} k_{2}(t, s) f_{2}(s, x(s)) d s
\end{aligned}
$$

which satisfy the following conditions:

(i) $k_{i}:[0,1] \times[0,1] \longrightarrow \mathbb{R}^{+}$and $f_{i}:[0,1] \times \mathbb{R} \longrightarrow$ $\mathbb{R}^{+}(i=1,2)$ are continuous. There exists a constant $D_{i} \in \mathbb{R}^{+}$ such that $\int_{0}^{1} k_{i}(t, s) d s \leq D_{i}$. And $\int_{0}^{1} k_{i}(t, s) f_{i}(s, x(s)) d s \leq K$, where $K \geq 0$ is a constant real number.

(ii) $\phi \in \Psi$ is nondecreasing and $\phi(t)<t$. There exists a constant $L_{i} \in[0,1)$ such that, for all $x, y \in X$,

$$
\left|f_{i}(t, x)\right|+\left|f_{i}(t, y)\right| \leq L_{i} \phi(|x|+|y|) \quad(i=1,2) .
$$

(iii) $2 K \cdot \max \left\{L_{1} D_{1}, L_{2} D_{2}\right\}<1$.

If $x \leq u, v \leq y$, or $u \leq x, y \leq v$, and there exist $x_{0}, y_{0} \in X$ such that $x_{0} \preceq T\left(x_{0}, y_{0}\right)$ and $T\left(y_{0}, x_{0}\right) \preceq y_{0}$, then $T$ has a coupled fixed point; i.e., (83) has a unique solution in $C(I)$. 
Proof. Denote $\sigma(x, y)=\sup _{t \in I}(|x(t)|+|y(t)|)$. Let $x \preceq u, v \preceq$ $y$, or $u \preceq x, y \preceq v$, and $x, y, u, v \in X$, and we have

$$
\begin{aligned}
& |T(x, y)(t)|+|T(u, v)(t)|=\left|\int_{0}^{1} k_{1}(t, s) f_{1}(s, x(s)) d s \int_{0}^{1} k_{2}(t, s) f_{2}(s, y(s)) d s\right| \\
& \quad+\left|\int_{0}^{1} k_{1}(t, s) f_{1}(s, u(s)) d s \int_{0}^{1} k_{2}(t, s) f_{2}(s, v(s)) d s\right| \\
& \quad \leq\left|\int_{0}^{1} k_{1}(t, s) f_{1}(s, x(s)) d s \int_{0}^{1} k_{2}(t, s)\left[\left|f_{2}(s, y(s))\right|+\left|f_{2}(s, v(s))\right|\right] d s\right| \\
& \quad+\left|\int_{0}^{1} k_{1}(t, s)\left[\left|f_{1}(s, x(s))\right|+\left|f_{1}(s, u(s))\right|\right] d s \int_{0}^{1} k_{2}(t, s) f_{2}(s, v(s)) d s\right| \leq K \cdot\left|\int_{0}^{1} k_{2}(t, s) L_{2}(\phi(\sigma(y, v))) d s\right|+K \\
& \quad \cdot\left|\int_{0}^{1} k_{1}(t, s) L_{1}(\phi(\sigma(u, x))) d s\right| \leq 2 K \cdot \max \left\{L_{1} D_{1}, L_{2} D_{2}\right\} \cdot \phi(\max \{\sigma(y, v), \sigma(u, x)\}) \\
& \quad \leq \phi(\max \{\sigma(y, v), \sigma(u, x), \sigma(T(x, y), u), \sigma(T(y, x), v)\})
\end{aligned}
$$

Hence, we get $\sigma(T(x, y), T(u, v)) \leq \phi(\max \{\sigma(y, v), \sigma(u$, $x), \sigma(T(x, y), u), \sigma(T(y, x), v)\})$. Then, all the conditions of Corollary 16 are satisfied with $g t=t$. Then $T$ has a coupled fixed point; i.e., (83) has a unique solution in $C(I)$.

\section{Data Availability}

No data were used to support this study.

\section{Conflicts of Interest}

The authors declare that they have no conflicts of interest.

\section{Acknowledgments}

The research is supported by the National Natural Science Foundation of China (11771198, 11701259, 11661053, 11461045, 11361042 , and 11071108).

\section{References}

[1] S. G. Matthews, "The topology of partial metric spaces," Research Report 222, Department of Computer Science, University of Warwick, 1992.

[2] S. G. Matthews, "Partial metric topology," in Proceedings of the 8th Summer Conference on General Topology and Applications, vol. 728, pp. 183-197, New York, NY, USA, 1994.

[3] W. Shatanawi and B. Samet, "On $(\psi, \phi)$-weakly contractive condition in partially ordered metric spaces," Computers \& Mathematics with Applications, vol. 62, no. 8, pp. 3204-3214, 2011.

[4] H. Aydi, M. Postolache, and W. Shatanawi, "Coupled fixed point results for $(\psi, \phi)$-weakly contractive mappings in ordered $G$ metric spaces," Computers \& Mathematics with Applications, vol. 63, no. 1, pp. 298-309, 2012.

[5] Z. Mustafa and B. Sims, "A new approach to generalized metric spaces," Journal of Nonlinear and Convex Analysis, vol. 7, no. 2, pp. 289-297, 2006.
[6] A. S. Saluja, M. S. Khan, P. K. Jhade, and B. Fisher, "Some fixed point theorems for mappings involving rational type expressions in partial metric spaces," Applied Mathematics ENotes, vol. 15, pp. 147-161, 2015.

[7] E. Karapinar, D. O’Regan, and B. Samet, "On the existence of fixed points that belong to the zero set of a certain function," Fixed Point Theory and Applications, vol. 2015, p. 152, 2015.

[8] H. Aydi, A. Felhi, and S. Sahmim, "Fixed points of multivalued nonself almost contractions in metric-like spaces," Mathematical Sciences, vol. 9, no. 2, pp. 103-108, 2015.

[9] T. G. Bhaskar and V. Lakshmikantham, "Fixed point theorems in partially ordered metric spaces and applications," Nonlinear Analysis: Theory, Methods \& Applications, vol. 65, no. 7, pp. 13791393, 2006.

[10] V. Lakshmikantham and L. Cirić, "Coupled fixed point theorems for nonlinear contractions in partially ordered metric spaces," Nonlinear Analysis. Theory, Methods \& Applications, vol. 70, no. 12, pp. 4341-4349, 2009.

[11] P. Hitzler and A. K. Seda, "Dislocated topologies," Journal of Electrical Engineering, vol. 51, no. 12, pp. 3-7, 2000.

[12] A. Amini-Harandi, "Metric-like spaces, partial metric spaces and fixed points," Fixed Point Theory and Applications, vol. 2012, p. 204, 2012.

[13] H. Aydi and E. Karapinar, "Fixed Point Results for Generalized $\alpha$ - $\nu$-contractions in metric-like spaces and applications," Electronic Journal of Differential Equations, vol. 2015, pp. 1-15, 2015.

[14] C. Zhu and $\mathrm{Z}$. Xu, "Inequalities and solution of an operator equation," Applied Mathematics Letters, vol. 21, no. 6, pp. 607611, 2008.

[15] C. Zhu, "Research on some problems for nonlinear operators," Nonlinear Analysis: Theory, Methods \& Applications, vol. 71, no. 10, pp. 4568-4571, 2009.

[16] Q. Li, C. Zhu, and S. Wang, "Bifurcation points and asymptotic bifurcation points of nonlinear operators in M-PN spaces," Nonlinear Analysis, vol. 71, no. 10, pp. 4960-4966, 2009.

[17] C. Zhu and C. Chen, "Calculations of random fixed point index," Journal of Mathematical Analysis and Applications, vol. 339, no. 2, pp. 839-844, 2008. 
[18] H. Aydi, E. Karapinar, and W. Shatanawi, "Tripled coincidence point results for generalized contractions in ordered generalized metric spaces," Fixed Point Theory and Applications, vol. 2012, article 101, 2012.

[19] H. Aydi, "Alpha-implicit contractive pair of mappings on quasi b-metric spaces and an application to integral equations," Journal of Nonlinear and Convex Analysis, vol. 17, no. 12, pp. 2417-2433, 2016.

[20] B. Hazarika, E. Karapinar, R. Arab, and M. Rabbani, "Metriclike spaces to prove existence of solution for nonlinear quadratic integral equation and numerical method to solve it," Journal of Computational and Applied Mathematics, vol. 328, pp. 302-313, 2018. 


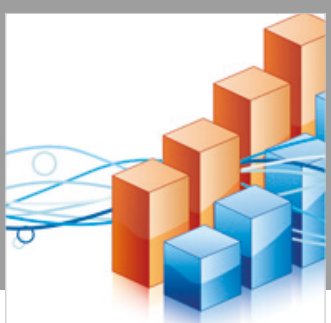

Advances in

Operations Research

\section{-n-m}
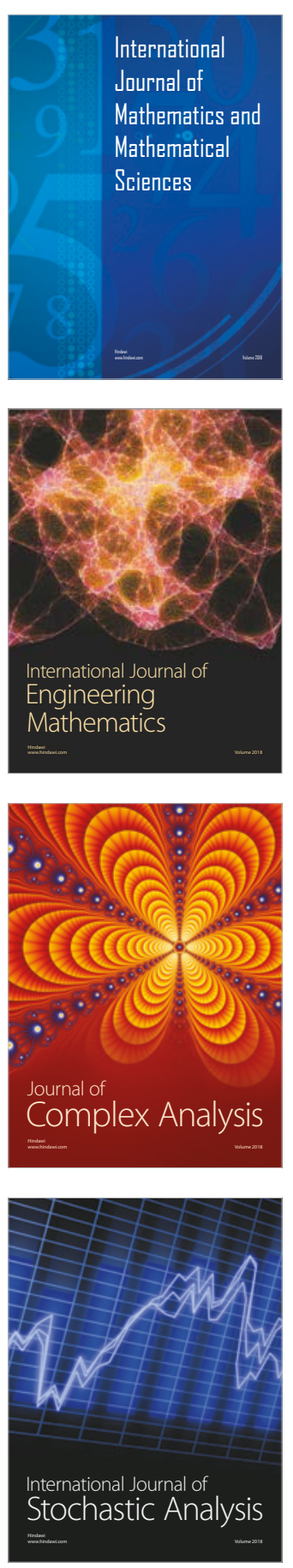
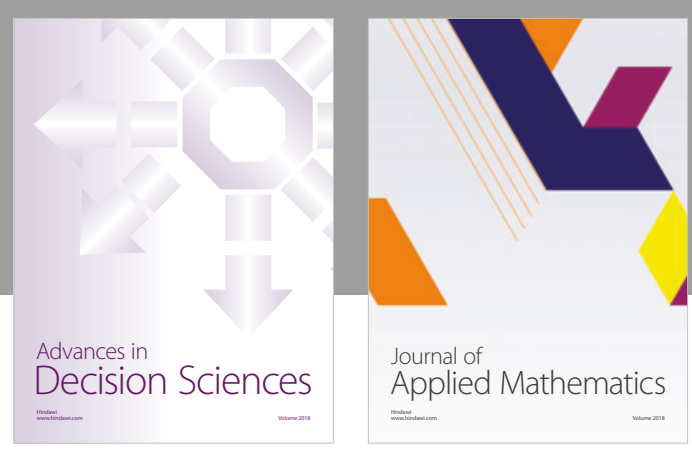

Journal of

Applied Mathematics
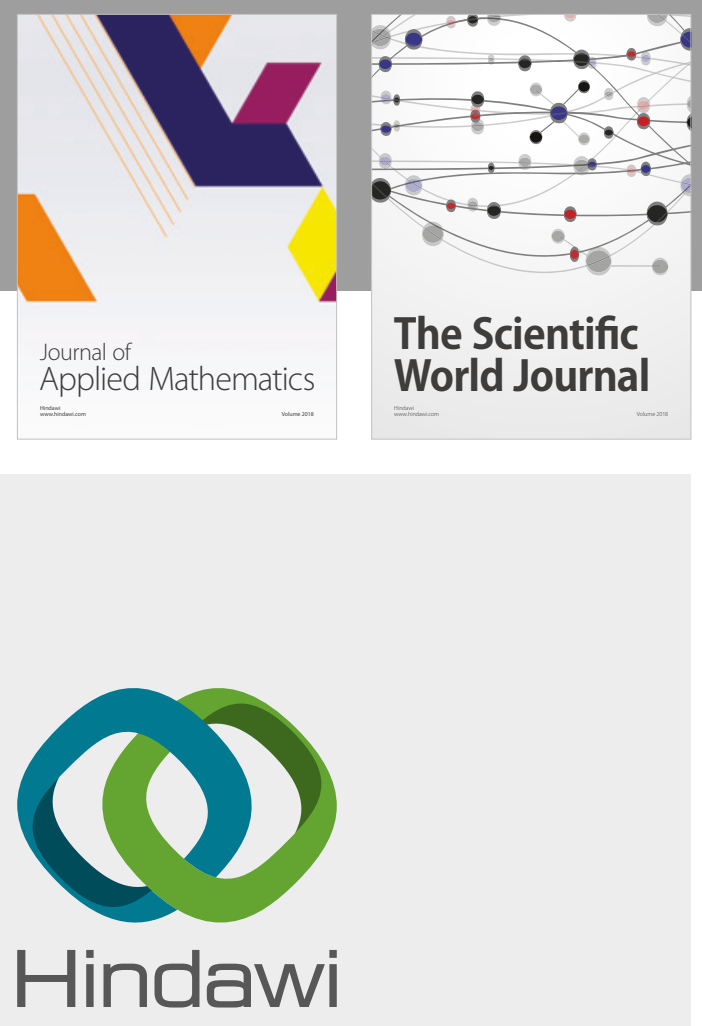

Submit your manuscripts at

www.hindawi.com

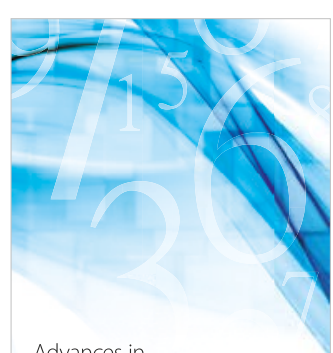

Advances in
Numerical Analysis
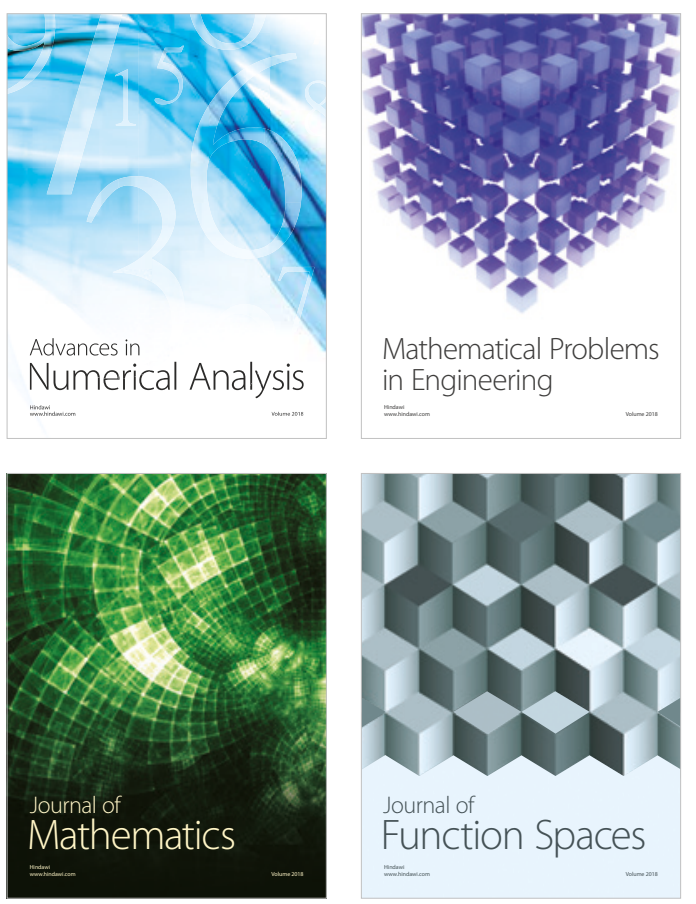

Mathematical Problems in Engineering

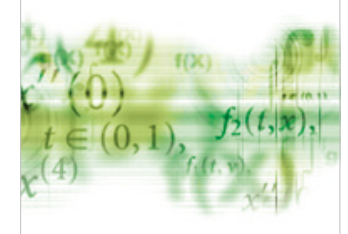

International Journal of

Differential Equations



Journal of

Function Spaces

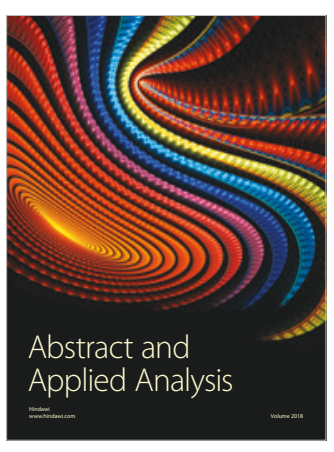

The Scientific

World Journal

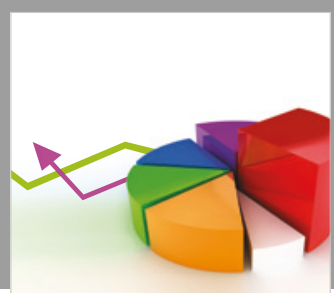

Journal of

Probability and Statistics
